\title{
Proximity-induced spin ordering at the interface between a ferromagnetic metal and a magnetic semiconductor
}

\author{
V. N. Men'shov, ${ }^{1,2}$ V. V. Tugushev,,$^{2,3,4}$ S. Caprara, ${ }^{1,5}$ and E. V. Chulkov ${ }^{1,3,4}$ \\ ${ }^{1}$ Donostia International Physics Center (DIPC), P. de Manuel Lardizabal 4, San Sebastián, Basque Country 20018, Spain \\ ${ }^{2}$ RRC Kurchatov Institute, Kurchatov Sqr. 1, 123182 Moscow, Russia \\ ${ }^{3}$ Departamento de Física de Materiales, Facultad de Ciencias Químicas, UPV/EHU, Apdo. 1072, \\ San Sebastián, Basque Country 20080, Spain \\ ${ }^{4}$ Centro de Física de Materiales (CFM), CSIC-UPV/EHU, Apdo. 1072, San Sebastián, Basque Country 20080, Spain \\ ${ }^{5}$ SMC-INFM-CNR. Dipartimento di Fisica, Università di Roma "La Sapienza," Piazzale Aldo Moro 2, 00185 Rome, Italy
}

(Received 17 February 2010; published 17 June 2010)

\begin{abstract}
We carry on a theoretical investigation of the conditions for the appearance and/or modification of spin ordering in a dilute magnetic semiconductor that is in contact with a ferromagnetic metal. We show that the magnetic proximity effect has a rather complex physical nature in this system. Allowing for the hybridization between the ferromagnetic metal and semiconductor electron states, we calculate the spin polarization and spin susceptibility of carriers in the semiconductor layer near the contact. The peculiar mechanism of indirect exchange coupling that occurs between local spins dissolved in the semiconductor host when a dilute magnetic semiconductor is in contact with a ferromagnetic metal is analyzed. The structure of the proximity-induced ordering of local moments in a dilute magnetic semiconductor is qualitatively described within a mean-field approach. On the basis of our results, we interpret the experimental data on Fe/(Ga,Mn)As and $\mathrm{Py} /(\mathrm{Ga}, \mathrm{Mn}) \mathrm{As}$ layered structures.
\end{abstract}

DOI: $10.1103 /$ PhysRevB.81.235212

PACS number(s): 73.40.Sx, 75.70.-i

\section{INTRODUCTION}

An important trend of investigation of the dilute magnetic semiconductors (DMSs) based on III-V (GaAs, GaSb) and IV ( $\mathrm{Si}, \mathrm{Ge})$ nonmagnetic semiconductors doped with magnetic $3 d$ transition-metal (TM) ( $\mathrm{Mn}, \mathrm{Fe}, \mathrm{Cr}$, and $\mathrm{Co}$ ) atoms is to obtain alloys that display electrically tunable ferromagnetism (FM) well above room temperature. However, it is still difficult to guess which of DMSs are most likely to display a high-temperature FM needed for spintronic applications. Although substantial progress has been made in experimental and theoretical studies of these materials, a deep understanding of the physics of DMSs has not been achieved.

It would seem that the manner to raise the Curie temperature $T_{c}$ in homogeneous (at the macroscales and nanoscales) DMSs, in which magnetic ions are more or less randomly distributed inside the semiconductor host, consists merely in increasing the concentration of TM ions. There are two sources of such an enhancement of ferromagnetic order in the alloy. ${ }^{1}$ The first is simply due to the rise of concentration of magnetic moments which is proportional to the concentration of TM ions in the DMS. The second arises from the fact that, according to the traditional point of view, an indirect exchange coupling between local moments dissolved inside the semiconductor host is mediated by the carriers of the host. The TM ions in the DMS play a twofold role: they carry the local moments and also provide the host with charge carriers. For example, when Mn substitutes for Ga in a GaAs lattice, it acts as an acceptor in the host, providing holes that mediate a ferromagnetic interaction between the local moments of the Mn ions. The strong correlation between the Curie temperature and the hole concentration $p$, approximated by an empirical relation $T_{c} \sim p^{1 / 3}$, has been established experimentally for the $\mathrm{Ga}_{1-x} \mathrm{Mn}_{x} \mathrm{As}$ alloys in a $0.02<x<0.085$ range of Mn content. ${ }^{2}$ However, the main unsolved problem concerns the state of the carriers in DMSs. The Ruderman-Kittel-Kasuya-Yoshida (RKKY)-type models of exchange imply that the carriers are itinerant and their chemical potential lies inside the valence or conduction band of the semiconductor host. Alternatively, the magnetic polaron models of exchange require that the carriers move in the impurity band and their chemical potential lies inside the gap of the semiconductor host.

Numerous experimental studies demonstrated that well above the solubility limit of TMs in a semiconductor host (of few atomic percents) it is hardly possible to achieve a homogeneous alloy. The incorporation of a large concentration of TM atoms is accompanied by macroscale and nanoscale fluctuations of the TM content of the alloy and the formation of inclusions of the thermodynamically stable phases (precipitates). As observed in the epitaxial growth of thin-film DMSs, a remarkable modulation of dopant density of different scales and various morphologies (from TM-rich small nanosized islands and droplets to long nanocolumns and micrometer-sized stripes) occurs. The presence of these inhomogeneities, which are usually not well controlled, strongly affects the crystal potential and the charge density in the material and is detrimental to the carrier mobility of most semiconductors. The structurally inhomogeneous DMSs often have relatively unconventional magnetic properties, including an unusual temperature dependence of the magnetization, transport, and optical characteristics, ascribed to the coexistence of paramagnetic and ferromagnetic regions and multiple competing exchange interactions. The clustering of impurities could locally increase the critical temperature but, as a rule, limits the achievable temperature of global ferromagnetic ordering $T_{c}{ }^{3}$ While achieving FM above room 
temperature in DMSs by improving the technology of preparation of well-controlled homogeneous alloys with traditional constituents or prospecting new materials remains a challenge, alternative ways to overcome this obstacle become very important.

The exchange coupling between magnetic ions can be remarkably increased in systems with artificially modulated doping, for example, the so-called digital magnetic alloys, due to the appearance of spin-polarized collective states of carriers (confinement states) ${ }^{4-9}$ A promising way to realize this is to synthesize heterostructures consisting of highly doped thin layers of TMs embedded into a semiconductor host. This is attractive because much higher local TM ions concentration can be obtained rather than that in a bulk random alloy. Following this idea Nazmul et al. ${ }^{10}$ have achieved a fairly high $T_{c}$ of about $250 \mathrm{~K}$ in $\mathrm{Mn} \delta$ doped GaAs/AlGaAs heterostructures. In our view, an advance in the field of digital magnetic alloys is connected with the exploitation of modern epitaxial techniques to produce structures that display a sharp profile of the metal $\delta$ layer along the growth direction.

One can suggest that the physical properties of DMSs will be strongly modified if prepared in the form of an ultrathin film on a metal substrate or sandwiched between metal layers. Recent measurements of magnetic order and interface coupling in a hybrid structure Fe/(Ga,Mn)As(100) have confirmed this suggestion. Maccherozzi et al. ${ }^{11}$ observed that induced magnetic order persists up to room temperature and extends over a DMS region as thick as $2 \mathrm{~nm}$ due to a robust exchange coupling at the interface between the (Ga,Mn)As layer and the Fe overlayer. It is evident that the spin order may be induced in otherwise paramagnetic systems (or may be enhanced in otherwise weak magnetic systems) owing to the close proximity to a strong ferromagnet. In agreement with Ref. 11 the magnetic behavior of the $\mathrm{Fe} /$ (Ga,Mn)As(100) structure is obviously a result of the magnetic proximity effect, when the wave function of spinpolarized states of the ferromagnetic metal (FMM) layer penetrates into the DMS layer. In such FMM/DMS hybrid structure, a strong magnetic order of a FMM with the Curie temperature well above room temperature could successfully be exploited to amplify the intrinsic Curie temperature $T_{c}$ of the DMS, thanks to the proximity effect. It is clear that the relevant magnetic order would be remarkably enhanced only near the interface with a FMM, in other words, it would be short ranged. Nevertheless, if the spin polarization penetrates from a FMM deeply enough into a DMS (according to Ref. 11 , in several nanometers at room temperature), the idea to bring a DMS in the close proximity of a strong FMM is highly fruitful for practical applications.

The goal of the present paper is to shed light on how magnetic proximity effect influences the exchange interaction between local magnetic moments of a DMS that is in contact with a FMM. Of course, in real FMM/DMS structures, the role of the interface in the emergence or modification of magnetic ordering in the semiconductor side cannot be solely attributed to the above mentioned "tunneling" mechanism of the magnetic proximity effect. The role of the interface is much more intricate and depends on its character and peculiarities. For example, in the case of the Fe/GaAs junction with Schottky barrier, interface localized states appear intrinsically within the barrier (see Ref. 12). In the Fe/Si structures, an intermediate layer of silicide forms at the interface due to unavoidable interdiffusion of the components. In addition, the boundary between the metal layer and the DMS layer can cause confinement of quasi-two-dimensional states of carriers in the semiconductor side of the hybrid structure. ${ }^{7}$ These peculiarities can have an impact on magnetic properties of the corresponding systems. However, in spite of their importance in specific situations, the question about the role of the tunneling mechanism of the magnetic proximity effect in FMM/DMS nanostructures is itself of a great interest.

Per se, the complex nature of spin-polarized tunneling through a metal/semiconductor interface was recognized rather long ago when dealing with the task of calculating magnetoresistance and spin diffusion for trilayers of ferromagnet/insulator/ferromagnet or ferromagnet/ semiconductor/ferromagnet types. It is known that, in such systems, near the interfaces, the effective-mass approximation is not valid. Therefore, various numerical methods need to be developed. Alternatively, one often applies simplified analytical schemes, where the tunneling probability (transmission coefficient) is determined by matching the wave function (and its derivative) obtained solving the Schrödinger equation with a semiphenomenological (as a rule, one-dimensional) potential at the interface between different materials. For example, in the model of Ref. 13, the free spin-polarized electrons tunnel through a rectangular barrier. In more refined quantum interference (electrooptical) model, ${ }^{14}$ Bruno introduced the concept of partial reflection coefficients for electron waves of different angle symmetry taking into account spin asymmetry of the reflections. Unfortunately, these schemes are of little use for solving our problem that consists in finding out the contribution to the energy of indirect exchange between the local moments of the DMS layer induced by the proximity to the FMM layer.

Let us briefly sketch the approach we use to evaluate the effect of the FMM/DMS contact on the magnetic ordering in DMS. Below we consider an interface between semi-infinite layers of FMM and DMS. The electron states of the DMS and FMM layers in the absence of the contact are assumed to be described on the basis of eigenstates of the bulk DMS, $\{\mathrm{S}\}$, and bulk FMM, $\{\mathrm{M}\}$, correspondingly. After the DMS layer contacts with the FMM layer, these states are perturbed by an interface potential. In general, the symmetry group of the interface potential does not coincide with the symmetry group of both the bulk DMS and FMM. This means, that in the DMS layer near the interface there are scattering processes both from the states $\{\mathrm{S}\}$ to the states $\{\mathrm{S}\}$ (intraband processes) and from the states $\{\mathrm{S}\}$ to the states $\{\mathrm{M}\}$ (interband processes). Specifying the interface potential by means of a set of phenomenological parameters, i.e., matrix elements of quasiparticle transitions between the states $\{\mathrm{S}\}$ and $\{\mathrm{M}\}$, one can evaluate the spin polarization and spin susceptibility of carriers in the DMS layer. From the methodological point of view, to perform this calculation, we involve the one- and two-particle Green's function, which contain both diagonal and off-diagonal components (in terms of the $\{S\}$ and $\{M\}$ 
states and with respect to spin indices). Knowing the spin polarization and spin susceptibility of carriers, one can calculate the contact-induced contribution to the energy of interaction between the local moments in the DMS layer.

The paper is organized as follows. In Sec. II, we discuss the model for a contact between FMM and DMS layers, which includes the hybridization of the $\{\mathbb{M}\}$ and $\{\mathrm{S}\}$ states at the FMM/DMS interface. In Sec. III, we consider the spin polarization of carriers in the semiconductor layer that is induced by the adjacent FMM layer and determine the dependence of the polarization on the distance from the interface. In Sec. IV the behavior of a single local moment (we also use in the text the equivalent term "local spin") under the influence of the carrier spin polarization in the DMS layer is described. In this section we also elucidate how the proximity of the FMM layer modifies the carrier mediated exchange coupling between local spins in the DMS layer. We derive the expression for the exchange energy of local spins dissolved in the DMS layer contacted the FMM layer. In Sec. $\mathrm{V}$, within the framework of an effective mean-field functional, we investigate the magnetization of the DMS layer as a function of the distance from the interface and temperature. The interpretation of experimental data on the Fe/(Ga,Mn)As (Ref. 11) and Py/(Ga,Mn)As (Ref. 15) layered systems, summary and concluding remarks are found in Sec. VI.

\section{SETUP OF THE PROBLEM AND MODEL HAMILTONIAN}

We consider the FMM/DMS system to be infinitely extended in all directions. Both the FMM and DMS layers are treated as semi-infinite slabs joined at the interface, the boundary between the constituent materials is implied to be perfectly flat. The system displays translational symmetry along the longitudinal direction in the $(x, y)$ interface plane located at $z=0$. Since the translational symmetry is broken along the $\mathbf{z}$ direction by the interface plane, it is convenient to use real-space coordinate representation. We write the Hamiltonian $H$ of the electron states of the FMM/DMS system in the form

$$
H=H_{S}+H_{M}+H_{I} .
$$

Here, the Hamiltonian $H_{S}$ describes the DMS layer, which occupies the half-space $z>0$,

$$
\begin{aligned}
H_{S}= & H_{0}+H_{l o c}=\int d \mathbf{r} \sum_{\alpha} \psi_{\alpha}^{+}(\mathbf{r}) \varepsilon(-i \nabla) \psi_{\alpha}(\mathbf{r}) \\
& +\sum_{i} \int d \mathbf{r} \sum_{\alpha, \beta} \psi_{\alpha}^{+}(\mathbf{r}) \kappa\left(\boldsymbol{\sigma}_{\alpha \beta} \cdot \mathbf{S}_{i}\right) \psi_{\beta}(\mathbf{r}) \delta\left(\mathbf{r}-\mathbf{R}_{i}\right),
\end{aligned}
$$

where the first term, $H_{0}$, is the kinetic energy of carriers in the pure semiconductor host (in the absence of the local spins), the second term, $H_{l o c}$, is the energy of interaction between the carriers and the local spins, and $\boldsymbol{\sigma}$ is the vector of the Pauli matrices. The restriction $|\kappa S| \ll W$ is imposed on the matrix element $\kappa$ of the exchange interaction of carriers with the local spins in Eq. (2) ( $W$ is an energy scale on the order of the bandwidth of the semiconductor).
It is necessary to make some additional assumptions. We assume that the local spin $\mathbf{S}_{i}$ at the site $\mathbf{R}_{i}=\left(X_{i}, Y_{i}, Z_{i}\right)$ of the semiconductor crystal lattice experiences a contact exchange interaction with carriers of the semiconductor host. To simplify the treatment, we shall describe the local spin $\mathbf{S}_{i}$ classically, regarding it as a magnetic moment denoted by the same symbol $\mathbf{S}_{i}$. All the spins are assumed to be of equal magnitude, $\left|\mathbf{S}_{i}\right|=S$. Formally speaking, our approach is correct in the case of a large spin, i.e., within a $1 / S$ expansion. Therefore one may hope that the classical approach will not introduce qualitative shortcomings in the estimate of the exchange interaction energy in DMSs. ${ }^{16}$

The magnetic TM ions within the DMS layer are assumed to be randomly dispersed with relatively low concentration up to several percent. Because of the relatively large distance between TM ions in DMS, the wave functions belonging to different ions have a negligibly small overlap, so the exchange coupling of two neighboring local spins in the DMS host is mediated by itinerant carriers. It should be noted that the spatial configuration of the magnetic order, which could establish within the DMS layer, is a priori uncertain. The arrangement of local spins is governed by exchange interactions of different origin and kind; some of them are intrinsic to DMSs while others are induced by the proximity to the FMM layer.

The second term in Eq. (1) is the Hamiltonian of the FMM layer, which occupies the half-space $z<0$,

$$
H_{M}=\int d \mathbf{r} \sum_{\alpha, \beta} \phi_{\alpha}^{+}(\mathbf{r})\left[E(-i \nabla) \delta_{\alpha \beta}+\left(\boldsymbol{\sigma}_{\alpha \beta} \cdot \boldsymbol{\Delta}\right)\right] \phi_{\beta}(\mathbf{r}),
$$

where $\Delta$ is the Stoner exchange splitting of the band states in the FMM bulk.

The metal and semiconductor quasiparticle states are scattered and mixed with each other and, in this manner, essentially modified (in comparison with the corresponding bulk states) near the FMM/DMS interface on the scale on the order of the lattice spacing. Moreover, a redistribution of charge and spin density is produced at the metal/ semiconductor boundary. Strictly speaking, it would be necessary to solve the rather intricate quantum-mechanical problem for electron states which experience the action of the self-consistent potential of the interface. Obviously, this task requires sophisticated numerical approaches but here we intend to make use of a simpler empirical method to obtain some qualitative estimates. Within the framework of this method, the electron structure of the constituent materials is assumed to be specified by the bulk parameters. In addition, the interface region must be phenomenologically described by means of an effective spin-dependent contact potential $\mathcal{V}(z)$. As pointed out above, the symmetry group of $\mathcal{V}(z)$ is, generally speaking, different from the symmetry groups of both the bulk metal and the bulk semiconductor. The operators $\psi_{\alpha}^{+}(\mathbf{r})\left[\psi_{\alpha}(\mathbf{r})\right]$ and $\phi_{\alpha}^{+}(\mathbf{r})\left[\phi_{\alpha}(\mathbf{r})\right]$ create (annihilate) a quasiparticle in a state belonging to the symmetry group of the bulk semiconductor $\{S\}$ or the bulk metal $\{M\}$ with spin projection $\alpha$ onto the quantization axis. These operators are defined far from the interface in the semiconductor layer $(z$ $>0)$ or in the metal layer $(z<0)$, where the potential $\mathcal{V}(z)$ 
vanishes. We introduce the term $H_{I}$, which describes in a phenomenological manner the effective Hamiltonian of quasiparticles at the interface,

$$
H_{I}=H_{s}+H_{m}+H_{s m}
$$

where

$$
\begin{gathered}
H_{s}=\int d \mathbf{r} \sum_{\alpha \beta} \psi_{\alpha}^{+}(\mathbf{r})\left[U_{s} \delta_{\alpha \beta}+\left(\boldsymbol{\Omega}_{s} \cdot \boldsymbol{\sigma}_{\alpha \beta}\right)\right] \psi_{\beta}(\mathbf{r}) \delta(z), \\
H_{m}=\int d \mathbf{r} \sum_{\alpha \beta} \phi_{\alpha}^{+}(\mathbf{r})\left[U_{m} \delta_{\alpha \beta}+\left(\boldsymbol{\Omega}_{m} \cdot \boldsymbol{\sigma}_{\alpha \beta}\right)\right] \phi_{\beta}(\mathbf{r}) \delta(z), \\
H_{s m}=\int d \mathbf{r} \sum_{\alpha \beta}\left\{\psi_{\alpha}^{+}(\mathbf{r})\left[U_{s m} \delta_{\alpha \beta}+\left(\boldsymbol{\Omega}_{s m} \cdot \boldsymbol{\sigma}_{\alpha \beta}\right)\right] \phi_{\beta}(\mathbf{r})+\phi_{\alpha}^{+}(\mathbf{r})\right. \\
\left.\quad \times\left[U_{m s} \delta_{\alpha \beta}+\left(\boldsymbol{\Omega}_{m s} \cdot \boldsymbol{\sigma}_{\alpha \beta}\right)\right] \psi_{\beta}(\mathbf{r})\right\} \delta(z)
\end{gathered}
$$

Here, we used a local approximation $\mathcal{V} \delta(z)$ for the potential $\mathcal{V}(z)$, all matrix elements of $\mathcal{V}$ are treated in our model as phenomenological parameters. The matrix elements $U_{s}, \boldsymbol{\Omega}_{s}$ and $U_{m}, \boldsymbol{\Omega}_{m}$ relate to the processes of potential and exchange scattering from the component $\mathcal{V}(z)$ without change of a symmetry of an eigenstate of the metal and semiconductor subsystem, respectively; while the matrix elements $U_{s m}, \Omega_{s m}$ and $U_{m s}, \boldsymbol{\Omega}_{m s}\left(U_{s m}=U_{m s}^{*}, \boldsymbol{\Omega}_{s m}=\boldsymbol{\Omega}_{m s}^{*}\right)$ are assigned to the scattering amplitude from the potential $\mathcal{V}(z)$ with transfer of quasiparticle from an eigenstate of symmetry group $\{\mathrm{S}\}$ to an eigenstate of symmetry group $\{\mathbb{M}\}$, and vice versa. The physical dimension of all the contact matrix elements equals an energy divided by an area.

In this work we are interested in the variation in charge and spin density caused by the redistribution of carriers in the DMS near the FMM/DMS interface over a tunneling length of few nanometers, i.e., much larger than the lattice parameters in bulk FMM and DMS. On the FMM side of the interface the local approximation for the potential $\mathcal{V}(z)$ is evident. It is assumed that the characteristic range of $\mathcal{V}(z)$ is small compared to the screening length $\lambda_{m}$ in the FMM, that is reasonable at all carrier concentrations, which exceed $10^{22} \mathrm{~cm}^{-3}$. On the DMS side of the interface the situation is not so simple. At relatively high carrier concentration, larger than $10^{19} \mathrm{~cm}^{-3}$, the carriers form a degenerate Fermi gas and the estimated screening length $\lambda_{s}$ does not exceed few angstroms, i.e., it is lesser than the tunneling length of the carriers. So, the local approximation seems reasonable. However, at relatively low carrier concentration, less than $10^{18} \mathrm{~cm}^{-3}$, the screening length $\lambda_{s}$ may formally be on the order of the tunneling length of the carriers. Apparently, such low carrier concentration is almost impossible in the bulk DMS at the TM concentration of few atomic percents, even in the case of an almost perfectly compensated material. The more realistic scenario is probable, when a wide region, strongly depleted with carriers (the so-called Schottky barrier), appears in the DMS side of the FMM/DMS interface due to the charge redistribution and the electron band bending near the interface. Since the thickness of the Schottky barrier can be as large as hundreds of nanometers, the local approximation for $\mathcal{V}(z)$ becomes formally incorrect. Never- theless, note that, for purely qualitative estimates, the longrange part of the potential $\mathcal{V}(z)$ may be simply included into the calculation through renormalization of the chemical potential of carriers in the DMS. In the following, we assume that the position of the Fermi level in the FMM layer defines the position of the chemical potential of carriers in the DMS layer. We also do not take into account the bending of electron bands in DMS near the FMM/DMS interface.

We consider our model at zero temperature, although the finite-temperature regime can, in principle, be accounted for by including the Fermi functions in the integrands of the expressions below. The free energy of the system can be formally written as

$$
F=-\frac{1}{\pi} \operatorname{Im} \int d \omega \operatorname{Tr} \ln G(\omega),
$$

where $G(\omega)=\left(\omega+i 0-H_{S}-H_{s}-H_{s m}\right)^{-1}$ is the Green's function of the DMS in the presence of the contact with the FMM layer, $\omega$ is the frequency. Following Ref. 16, we express the quantity $\ln G(\omega)$ in terms of the components of the Green's function of the pure semiconductor host (without the dissolved local spins) coupled to the FMM layer, $g(\omega)=(\omega$ $\left.+i 0-H_{0}-H_{s}-H_{s m}\right)^{-1}$. The function comprises diagonal $\left(g^{d}\right)$ and off-diagonal $\left(g^{o d}\right)$ components with respect to the indexes of the local-spin sites $\mathbf{R}_{i}$. The symbolic form of the corresponding expression is

$$
\ln G=\ln g-\ln \left(1-g^{d} \mathbb{K}\right)+\sum_{n=1}^{\infty} \frac{1}{n}\left[g^{o d} t\right]^{n},
$$

where the symbol $t$ denotes the single-site scattering matrix that describes the multiple scattering of electrons on the local spin placed at the point $\mathbf{R}_{i}$,

$$
t=\mathbb{K}\left[\mathbb{I}-g^{d} \mathbb{K}\right]^{-1} .
$$

By taking into account the spin indexes, the matrix $\mathbb{K}$ is written as $\mathbb{K}_{\alpha \beta}\left(\mathbf{R}_{i}\right)=\kappa\left(\sigma_{\alpha \beta} \cdot \mathbf{S}_{i}\right)$.

According to Eq. (7), the free energy, Eq. (6), may be divided into three contributions,

$$
F=F_{0}+F_{d}+F_{o d} .
$$

The term $F_{0}$ is the free energy of the semiconductor host in the presence of the contact, which is independent of the interaction between electrons and local spins. The last two terms in Eq. (8) represent the part of the free energy caused by the exchange interaction of carriers with local spins dispersed in the DMS layer. The site-diagonal term $F_{d}$ appears in the single-site approximation, whereas the site-offdiagonal term $F_{o d}$ is related to the interference of the states belonging to different local-spin sites. Since we are interested in the situation of a strongly diluted alloy in the DMS layer, the terms up to the second order of $g^{\text {od }}$ will be retained to calculate the energy contribution $F_{o d}$.

\section{CONTACT-INDUCED SPIN POLARIZATION OF CARRIERS IN THE SEMICONDUCTOR LAYER}

The effect of the metal/semiconductor interface on redistribution of the charge and spin densities in the semiconduc- 
tor layer can be evaluated from the variation in the Green's function of the semiconductor layer due to the interface potential treated as a perturbation. Consistently with our assumptions, we write down the Green's function of the semiconductor layer in the coordinate representation as

$$
g_{\alpha \beta}\left(\mathbf{r} ; \mathbf{r}^{\prime} ; \omega\right)=g^{0}\left(\mathbf{r} ; \mathbf{r}^{\prime} ; \omega\right) \delta_{\alpha \beta}+\Xi_{\alpha \beta}\left(\mathbf{r} ; \mathbf{r}^{\prime} ; \omega\right),
$$

where $g^{0}$ is the Green's function of the semiconductor bulk (in the absence of the metallic layer and local spins) associated with the Hamiltonian $H_{0}$. Evaluating the variation $\Xi$, we restrict ourselves to the lowest order in the perturbation, Eqs. (3) and (5), i.e., the first order in the parameters $U_{s}$ and $\boldsymbol{\Omega}_{s}$ and the second order in the parameters $U_{s m}$ and $\boldsymbol{\Omega}_{s m}$. Within such an approximation, we arrive to the following expressions:

$$
\begin{aligned}
& \Xi_{\alpha \beta}\left(\mathbf{r} ; \mathbf{r}^{\prime} ; \omega\right)=\Lambda_{\alpha \beta}\left(\mathbf{r} ; \mathbf{r}^{\prime} ; \omega\right)+\Pi_{\alpha \beta}\left(\mathbf{r} ; \mathbf{r}^{\prime} ; \omega\right) \\
& \Lambda_{\alpha \beta}\left(\mathbf{r} ; \mathbf{r}^{\prime} ; \omega\right)=\left[U_{s} \delta_{\alpha \beta}+\left(\boldsymbol{\Omega}_{s} \cdot \sigma_{\alpha \beta}\right)\right] \int d \boldsymbol{\rho}_{1} \\
& \times g^{0}\left(\boldsymbol{\rho}, z ; \boldsymbol{\rho}_{1}, 0 ; \omega\right) g^{0}\left(\boldsymbol{\rho}_{1}, 0 ; \boldsymbol{\rho}^{\prime}, z^{\prime} ; \omega\right) \\
& \Pi_{\alpha \beta}\left(\mathbf{r} ; \mathbf{r}^{\prime} ; \omega\right)= \sum_{\gamma, \delta} \int d \boldsymbol{\rho}_{1} d \boldsymbol{\rho}_{2} g^{0}\left(\boldsymbol{\rho}, z ; \boldsymbol{\rho}_{1}, 0 ; \omega\right) \times\left[U_{s m} \delta_{\alpha \gamma}\right. \\
&\left.+\left(\boldsymbol{\Omega}_{s m} \cdot \boldsymbol{\sigma}_{\alpha \gamma}\right)\right] \times \mathcal{G}_{\gamma \delta}\left(\boldsymbol{\rho}_{1}, 0 ; \rho_{2}, 0 ; \omega\right) \\
& \times\left[U_{m s} \delta_{\delta \beta}+\left(\Omega_{m s} \cdot \boldsymbol{\sigma}_{\delta \beta}\right)\right] \\
& \times g^{0}\left(\boldsymbol{\rho}_{2}, 0 ; \rho^{\prime}, z^{\prime} ; \omega\right)
\end{aligned}
$$

where $\mathcal{G}_{\alpha \beta}\left(\mathbf{r} ; \mathbf{r}^{\prime} ; \omega\right)$ is the Green's function of the bulk FMM, i.e., $\mathcal{G}(\omega)=\left(\omega+i 0-H_{M}\right)^{-1}$ and $\boldsymbol{\rho}=(x, y)$ is the coordinate vector along the interface plane.

Thus, in Eq. (9), we express the Green's function of the semiconductor layer adjacent to the metal layer by means of the Green's functions $g^{0}$ and $\mathcal{G}$ associated with the bulk semiconductor and the bulk metal, respectively. The component $\Lambda_{\alpha \beta}\left(\mathbf{r} ; \mathbf{r}^{\prime} ; \omega\right)$ results from the intraband electron excitations due to the interface in the semiconductor while the component $\Pi_{\alpha \beta}\left(\mathbf{r} ; \mathbf{r}^{\prime} ; \omega\right)$ results from the interband excitations between the metal and semiconductor states. Thereby, as seen from Eqs. (11) and (12), we formally deal with the task of the perturbation of the electron density in the infinite bulk semiconductor by the frequency- and spin-dependent effective potential, which is nonlocal. However, only the half-space $z>0$ contributes to the energy, Eqs. (6) and (8).

For the sake of definiteness, we suppose that the Fermi level of the system $\mu$ lies near the bottom of the conduction band of the semiconductor. Thereby, we use the simplified model in which the electron states of this semiconductor band are mainly involved in the processes of the hybridization and scattering at the interface while the contribution of other energy bands (distant from the Fermi level) is ignored. We adopt henceforth the simplest form for the energy spectrum of the semiconductor host,

$$
\varepsilon(\mathbf{k})=\frac{\mathbf{k}^{2}}{2 m},
$$

where $m$ is the effective quasiparticle mass near the band bottom located at zero energy.

Within the adopted effective-mass approximation, the Green's function describing the propagation of an electron with energy $\omega>0$ between the points $\mathbf{r}$ and $\mathbf{r}^{\prime}$ in the semiconductor host reads,

$$
g^{0}\left(\mathbf{r} ; \mathbf{r}^{\prime} ; \omega\right)=-\frac{m a^{3}}{2 \pi} \frac{\exp \left(i \sqrt{2 m \omega}\left|\mathbf{r}-\mathbf{r}^{\prime}\right|\right)}{\left|\mathbf{r}-\mathbf{r}^{\prime}\right|},
$$

where $a$ is the semiconductor lattice spacing. One can use Eq. (13) for electrons with negative energy $\omega<0$, if one substitutes $-\sqrt{|\omega|}$ for $i \sqrt{\omega}$.

For the FM metal host we adopt the simplest form of the spectrum with two spin-split subbands,

$$
E^{( \pm)}(\mathbf{k})=E(\mathbf{k}) \pm \Delta=\frac{\mathbf{k}^{2}}{2 \tilde{m}}-E_{0} \pm \Delta,
$$

where $\tilde{m}$ is the effective mass of electrons near the minimum of the subbands. The quasiparticle effective mass in the semiconductor is noticeably smaller than that in the $d$ band of the metal, $\tilde{m}>m$. The energies $E_{0}^{( \pm)}=E_{0} \mp \Delta>0$ determine the lower edges of the minority-spin and majority-spin bands, located at $-E_{0}^{( \pm)}$, respectively, $\Delta$ is the energy of exchange splitting in the ferromagnetic metal. In what follows, to simplify the analytical treatment, we shall regard the potential step at the interface $E_{0}$ as largest energy parameter in our model. Of course, in the case of the Fe/(Ga,Mn)As(100) junction, the values $E_{0}$ and $\Delta$ are likely to be of the same order of magnitude. In this case, the exchange mechanism discussed below, would coexist with the usual mechanism ${ }^{14}$ associated with the penetration of the wave function of the metal into the semiconductor below the potential barrier, close enough to the interface. Anyway, due to the exponential fall-off of the latter, our mechanism would be the dominant one at larger distances from the interface.

The Green's function of the FM metal host is given by

$$
\begin{aligned}
\mathcal{G}_{\alpha \beta}\left(\mathbf{r}, \mathbf{r}^{\prime}, \omega\right)= & \frac{1}{2} \mathcal{G}^{(+)}\left(\mathbf{r}, \mathbf{r}^{\prime}, \omega\right)\left[\delta_{\alpha \beta}-\left(\mathbf{n} \cdot \boldsymbol{\sigma}_{\alpha \beta}\right)\right] \\
& +\frac{1}{2} \mathcal{G}^{(-)}\left(\mathbf{r}, \mathbf{r}^{\prime}, \omega\right)\left[\delta_{\alpha \beta}+\left(\mathbf{n} \cdot \boldsymbol{\sigma}_{\alpha \beta}\right)\right],
\end{aligned}
$$

where

$$
\mathcal{G}^{( \pm)}\left(\mathbf{r} ; \mathbf{r}^{\prime} ; \omega\right)=-\frac{\tilde{m} \tilde{a}^{3}}{2 \pi} \frac{\exp \left(i \sqrt{2 \tilde{m}\left[\omega+E_{0}^{( \pm)}\right]}\left|\mathbf{r}-\mathbf{r}^{\prime}\right|\right)}{\left|\mathbf{r}-\mathbf{r}^{\prime}\right|}
$$

and $\mathbf{n}=(\boldsymbol{\Delta} / \Delta)$ is the unit vector oriented along the direction of the magnetization of the FMM layer, $\tilde{a}$ is the metal lattice spacing.

Such assumptions regarding the electron structure of the semiconductor and metal subsystems do not significantly restrict the generality of our discussion with the advantage of leading to analytical expressions that provide an evident physical meaning of our results. 
Having introduced the Green's function for disconnected bulk subsystems, we now proceed to the explicit calculation of the variation in the Green's function of the semiconductor layer caused by the adjacent FMM layer. Let us represent the spin structure of the components, Eqs. (11) and (12), in the following form:

$$
\begin{aligned}
& \Lambda_{\alpha \beta}\left(\mathbf{r} ; \mathbf{r}^{\prime} ; \omega\right)=\widetilde{\Lambda}\left(\mathbf{r} ; \mathbf{r}^{\prime} ; \omega\right)\left[U_{s} \delta_{\alpha \beta}+\left(\boldsymbol{\Omega}_{s} \cdot \boldsymbol{\sigma}_{\alpha \beta}\right)\right], \\
& \Pi_{\alpha \beta}\left(\mathbf{r} ; \mathbf{r}^{\prime} ; \omega\right)=\widetilde{\Pi}^{(+)}\left(\mathbf{r} ; \mathbf{r}^{\prime} ; \omega\right) \Upsilon_{\alpha \beta}^{(+)}+\widetilde{\Pi}^{(-)}\left(\mathbf{r} ; \mathbf{r}^{\prime} ; \omega\right) \Upsilon_{\alpha \beta}^{(-)}, \\
& \Upsilon_{\alpha \beta}^{( \pm)}=\left[U_{s m} U_{m s}\right. \\
&\left.+\left(\Omega_{s m} \cdot \boldsymbol{\Omega}_{m s}\right) \mp U_{s m}\left(\mathbf{n} \cdot \boldsymbol{\Omega}_{m s}\right) \mp U_{m s}\left(\mathbf{n} \cdot \Omega_{s m}\right)\right] \delta_{\alpha \beta} \\
& \quad \mp\left[U_{s m} U_{m s}-\left(\boldsymbol{\Omega}_{s m} \cdot \Omega_{m s}\right)\right]\left(\mathbf{n} \cdot \boldsymbol{\sigma}_{\alpha \beta}\right) \\
&+\left[U_{s m} \mp\left(\mathbf{n} \cdot \Omega_{s m}\right)\right]\left(\boldsymbol{\Omega}_{m s} \cdot \boldsymbol{\sigma}_{\alpha \beta}\right)+\left[U_{m s} \mp\left(\mathbf{n} \cdot \Omega_{m s}\right)\right] \\
& \quad \times\left(\boldsymbol{\Omega}_{s m} \cdot \boldsymbol{\sigma}_{\alpha \beta}\right) \pm i U_{s m}\left(\boldsymbol{\sigma}_{\alpha \beta} \cdot[\mathbf{n}\right. \\
&\left.\left.\times \Omega_{m s}\right]\right) \mp i U_{m s}\left(\boldsymbol{\sigma}_{\alpha \beta} \cdot\left[\mathbf{n} \times \Omega_{s m}\right]\right) .
\end{aligned}
$$

We write the variation in the Green's function of a semiconductor $\Xi_{\alpha \beta}\left(\mathbf{r} ; \mathbf{r}^{\prime} ; \omega\right)$, Eq. (10), at equal space arguments, $\mathbf{r}=\mathbf{r}^{\prime}$. As it will be seen below, in the context of the problem under consideration, we do not need more elaborate information on this quantity. The spin-dependent density of states is the relevant quantity to elucidate how the contact influences the polarization of the semiconductor layer.

The spatial change in the Green's function of the semiconductor caused by the intraband processes at the interface is described by the following distribution:

$$
\begin{gathered}
\tilde{\Lambda}(\mathbf{r} ; \mathbf{r} ; \omega>0)=-\frac{m^{2} a^{6}}{2 \pi}[\operatorname{ci}(2 \sqrt{2 m \omega} z)+i \operatorname{si}(2 \sqrt{2 m \omega} z)], \\
\tilde{\Lambda}(\mathbf{r} ; \mathbf{r} ; \omega<0)=\frac{m^{2} a^{6}}{2 \pi} E_{1}(2 \sqrt{2 m|\omega|} z)
\end{gathered}
$$

For the spatial change in the Green's function of the semiconductor caused by the interband processes at the interface, using the Erdélyi lemma and omitting terms proportional to $E_{0}^{-3 / 2}$ (under the condition $E_{0}^{( \pm)} \gg|\omega|$ ), one arrives at the following distribution:

$$
\begin{aligned}
& \widetilde{\Pi}^{( \pm)}(\mathbf{r} ; \mathbf{r} ; \omega>0)=-\frac{m^{2} a^{6} \sqrt{\tilde{m}} \widetilde{a}^{3}}{4 \pi \sqrt{2 E_{0}^{( \pm)}}} \\
& \times[\operatorname{si}(2 \sqrt{2 m \omega} z)-i \operatorname{ci}(2 \sqrt{2 m \omega} z)], \\
& \widetilde{\Pi}^{( \pm)}(\mathbf{r} ; \mathbf{r} ; \omega<0)=-\frac{i m^{2} a^{6} \sqrt{\tilde{m}} \widetilde{a}^{3}}{4 \pi \sqrt{2 E_{0}^{( \pm)}}} E_{1}(2 \sqrt{2 m|\omega|} z) .
\end{aligned}
$$

Above, to distinguish between the expressions for the states with the positive energy and with negative energy, we label the former by $\omega>0$ and the latter by $\omega<0$. We utilize the standard notation for the special functions,

$$
\operatorname{si}(\mathrm{s})=-\int_{\varsigma}^{\infty} d t \frac{\sin t}{t}
$$

and

$$
\operatorname{ci}(\mathrm{s})=-\int_{\mathrm{s}}^{\infty} d t \frac{\cos t}{t}
$$

are the sine and cosine integrals, respectively,

$$
E_{1}(\mathrm{~s})=\int_{\varsigma}^{\infty} d t \frac{\exp (-t)}{t}
$$

is the exponential integral. ${ }^{17}$

The expression, Eqs. (16) and (17), are obtained under the condition $|\omega| \ll E_{0}$. Far from the point $\omega=0$, the value of $\widetilde{\Pi}^{( \pm)}(\mathbf{r} ; \mathbf{r} ; \omega)$ decays rapidly and becomes relatively small when $\omega \geq-E_{0}^{( \pm)}$and the value $2 \sqrt{2 m E_{0}^{( \pm)}} z$ is rather larger than unity,

$$
\tilde{\Pi}^{( \pm)}(\mathbf{r} ; \mathbf{r} ; \omega<0) \sim \sqrt[4]{\frac{E_{0}^{( \pm)}+\omega}{\left(E_{0}^{( \pm)}\right)^{3}}} \exp \left(-2 \sqrt{2 m E_{0}^{( \pm)}} z\right) .
$$

We have now all the ingredients that are needed to determine the spin polarization of carriers in the semiconductor layer due to the contact with the FM metallic layer. The spin polarization at the point $\mathbf{r}(z>0)$ is given by

$$
\mathbf{m}(\mathbf{r})=\operatorname{Im} \int^{\mu} \frac{d \omega}{\pi} \sum_{\alpha \beta} \boldsymbol{\sigma}_{\alpha \beta} \Xi_{\beta \alpha}(\mathbf{r}, \mathbf{r}, \omega) .
$$

In the spirit of the task setting, the semiconductor layer is assumed to be heavily doped, therefore the Fermi level of the system $\mu$ is placed within the semiconductor band, $\mu>0$. The applicability of the effective mass method requires $\mu$ to be small as compared with both the metal and the semiconductor bandwidth: $\mu \ll\left(E_{0}, W\right)$. On the other hand, of course, the size of the perturbing interface potential is not large on the scale of the bandwidths.

We express the dependence of the spin polarization of the carriers in the semiconductor layer [Eq. (18)] as a function of the variable $z$ and of the parameter $\mu$ in the additive form

$$
\mathbf{m}(z, \mu)=\mathbf{m}^{(\Lambda)}(z, \mu)+\mathbf{m}^{(\Pi)}(z, \mu) .
$$

Taking into account Eqs. (14) and (15), after integration with respect to Eq. (18), one obtains for the spin-polarization component originated from the intraband processes the following explicit expression:

$$
\begin{gathered}
\mathbf{m}^{(\Lambda)}(z, \mu)=-\boldsymbol{\Omega}_{s}\left(\frac{m a^{3}}{\pi}\right)^{2} \mu \phi\left(2 k_{F} z\right), \\
\phi(\xi)=\operatorname{si}(\xi)+\frac{\cos \xi}{\xi}-\frac{\sin \xi}{\xi^{2}},
\end{gathered}
$$

where $k_{F}$ is the Fermi momentum in the semiconductor layer, $k_{F}=\sqrt{2 m \mu}$, and $\xi=2 k_{F} z$ is a dimensionless quantity. In the asymptotic limit $2 k_{F} z \gg 1$, the range function $\phi$ exhibits damped oscillations approaching the behavior 


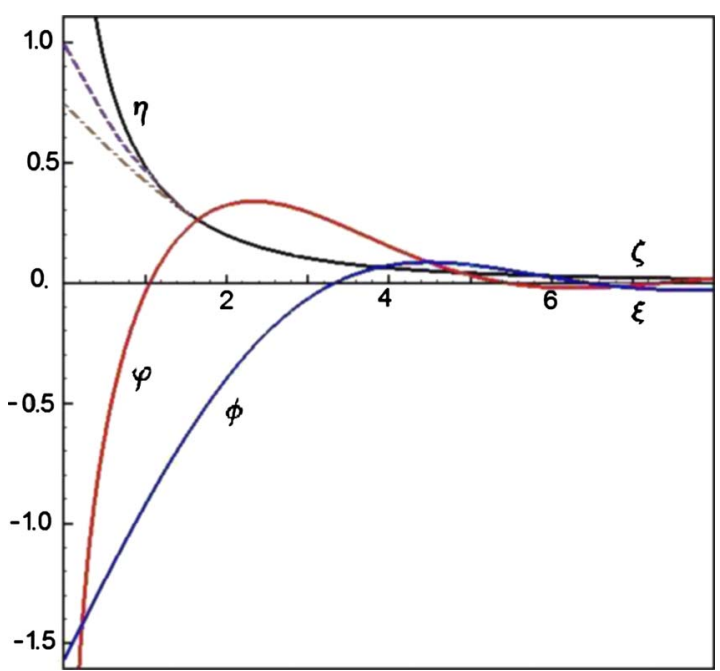

FIG. 1. (Color online) Range functions vs the dimensionless coordinates. The functions $\phi(\xi), \varphi(\xi)$, and $\eta(\zeta)$ are depicted by the lines marked with the corresponding labels. $\xi=2 k_{F} z$ and $\zeta=2 m E_{0} z$. The function $\eta(\zeta)$ is represented by the dashed line for $\tilde{m} / m=9$ and by the point-dashed line for $\tilde{m} / m=1$, respectively. Its asymptotic behavior, Eq. (25), is depicted by the solid line.

$$
\phi(\xi) \simeq \frac{2}{\xi^{2}}\left[\sin \xi-\frac{\cos \xi}{\xi}\right]
$$

In the opposite case $2 k_{F} z \ll 1$, we find

$$
\phi(\xi) \simeq-\frac{\pi}{2}+\frac{2 \xi}{3}
$$

The range function, Eq. (21), is depicted in Fig. 1.

Taking into account Eqs. (16) and (17), after integration in Eq. (18), one obtains for the spin-polarization component originated from the interband processes the following expression:

$$
\mathbf{m}^{(\Pi)}(z, \mu)=\boldsymbol{\Omega}_{h}\left(\frac{m a^{3}}{\pi}\right)^{2}\left[\mu \varphi\left(2 k_{F} z\right)-E_{0} \eta\left(2 \sqrt{2 m E_{0}} z\right)\right],
$$

$$
\begin{aligned}
\boldsymbol{\Omega}_{h}= & \frac{\sqrt{\tilde{m}} \widetilde{a}^{3}}{\sqrt{2 E_{0}}}\left(U_{s m} \boldsymbol{\Omega}_{m s}+U_{m s} \boldsymbol{\Omega}_{s m}+\frac{\Delta}{2 E_{0}}\left\{\left[U_{s m} U_{m s}\right.\right.\right. \\
& \left.-\left(\boldsymbol{\Omega}_{s m} \cdot \boldsymbol{\Omega}_{m s}\right)\right] \mathbf{n}+\left(\mathbf{n} \cdot \boldsymbol{\Omega}_{s m}\right) \boldsymbol{\Omega}_{m s}+\left(\mathbf{n} \cdot \boldsymbol{\Omega}_{m s}\right) \boldsymbol{\Omega}_{s m} \\
& \left.\left.-i U_{s m}\left[\mathbf{n} \times \boldsymbol{\Omega}_{m s}\right]+i U_{m s}\left[\mathbf{n} \times \boldsymbol{\Omega}_{s m}\right]\right\}\right) .
\end{aligned}
$$

The exchange splitting energy is considered to be small compared to the bandwidth, i.e., $\Delta \ll E_{0}$. By keeping the terms up to first order in $\Delta / E_{0}$, we have obtained Eq. (23).

In the right part of Eq. (22), in the square brackets, the first term, $\sim \mu$, is derived from the integration over the positive frequency $\omega$. The corresponding range function reads

$$
\varphi(\xi)=\operatorname{ci}(\xi)-\frac{\sin \xi}{\xi}+\frac{1-\cos \xi}{\xi^{2}} .
$$

The second term, $\sim E_{0}$, is produced by the integration from $\omega=-E_{0}$ to $\omega=0$. The range function $\eta(\zeta)$ appears to be a complicated function of the variable $z$ and the energy spectrum parameters $E_{0}$ and $m / \tilde{m}$. Under the condition $2 \sqrt{2 m E_{0}} z \gg 1$, the narrow region $0>\omega>-\nu\left(\nu \ll E_{0}\right)$ mainly contributes to the integral, and one may use the approximation

$$
\eta(\zeta)=E_{1}(\zeta)+\frac{1-(1+\zeta) \exp (-\zeta)}{\zeta^{2}} .
$$

Note that the function $\eta(\zeta)$ does not change sign and has the asymptotic behavior $\eta(\zeta) \simeq \zeta^{-2}$ when the dimensionless parameter $\zeta=2 m E_{0} z$ tends to infinity. Therefore, at large distance from the interface, when $2 k_{F} z \gg 1$, the polarization component $\mathbf{m}^{(\Pi)}(z)$ behaves as

$$
\mathbf{m}^{(\Pi)}(z, \mu) \simeq-\boldsymbol{\Omega}_{h}\left(\frac{m a^{3}}{\pi}\right)^{2} \frac{2 \mu}{\xi^{2}}\left[\cos \xi+\frac{\sin \xi}{\xi}\right],
$$

where $\xi=2 k_{F} z$.

When the arguments approach zero, the range function $\varphi(\xi)$ diverges logarithmically, while the function $\eta(\zeta)$ reaches a finite value, $\eta(\zeta \rightarrow 0) \rightarrow \eta_{0}$. At small $2 k_{F} z$ and $2 \sqrt{2 m E_{0}} z$, we find

$$
\mathbf{m}^{(\Pi)}(z, \mu) \simeq \boldsymbol{\Omega}_{h}\left(\frac{m a^{3}}{\pi}\right)^{2}\left[\mu \ln \left(2 k_{F} z\right)-E_{0} \eta_{0}\right],
$$

where, under reasonable assumptions for the effective-mass ratio $m / \tilde{m}$, the value $\eta_{0}$ is on the order of unity. It is interesting to remark that, if the Fermi level is rather low, namely, $\left|\mu \ln \left(2 k_{F} z\right)\right|<E_{0}$, the interface hybridization with the metal states, the energy levels of which are placed inside the semiconductor gap, gives rise mainly to the formation of the polarization $\mathbf{m}^{(\Pi)}(z, \mu)$, Eq. (26). It is important not to forget that our results are meaningful over distances (measured from the nominal boundary, $z=0$ ) which significantly exceed the characteristic length (on the order of the semiconductor lattice spacing) of the contact potential. Moreover, the effective-mass approximation is not correct in the semiconductor side near the interface within the region $z$ $\lesssim(\sqrt{m W})^{-1}$.

The behavior of the range functions $\varphi(\xi)$ and $\eta(\zeta)$ is shown in Fig. 1.

It is interesting to note that $\int_{0}^{\infty} d \xi \phi(\xi)=-2, \int_{0}^{\infty} d \xi \varphi(\xi)=0$, and $\int_{0}^{\infty} d \zeta \eta(\zeta)=2$ [making use of the approximation Eq. (25) for $\eta(\zeta)$ ], consequently, the net spin polarization of the DMS layer, $\boldsymbol{\nu}=\int_{0}^{\infty} d z \mathbf{m}(z)$, is

$$
\boldsymbol{\nu}=\left(\frac{m a^{3}}{\pi}\right)^{2}\left(\boldsymbol{\Omega}_{s} \sqrt{\frac{\mu}{2 m}}-\boldsymbol{\Omega}_{h} \sqrt{\frac{E_{0}}{2 m}}\right) .
$$

In principle, we could sum up an infinite perturbation series in the matrix elements of the contact potential and obtain an exact expression for the variation $\Xi_{\alpha \beta}\left(\mathbf{r} ; \mathbf{r}^{\prime} ; \omega\right)$ in Eq. (9) corresponding to the full $t$ matrix, in analogy with the results of Ref. 8. The corresponding calculations allow us to esti- 
mate the domain of applicability of the approximations used above: $a^{3}\left(\left|U_{s}\right|,\left|\Omega_{s}\right|\right) \sqrt{m} \ll \sqrt{\mu}$ and $a^{3} \widetilde{a}^{3} \sqrt{m \tilde{m}}\left(\left|U_{s m}\right|,\left|\Omega_{s m}\right|\right)^{2}$ $\ll \sqrt{2 E_{0} \mu}$.

We can assume that the vector $\boldsymbol{\Omega}_{s}$ is proportional to the magnetization of the FMM layer $\boldsymbol{\Omega}_{s}=J \boldsymbol{\Delta}$. Strictly speaking, the same assumption does not hold for the vector $\boldsymbol{\Omega}_{h}$, Eq. (23). Hence, in the general case, the total spin polarization in the semiconductor, m, Eq. (19), which is likely to arise not only due to the imbalance of filling of the majority-spin and minority-spin subbands in the FMM layer $(\sim \boldsymbol{\Delta})$ but also due to the fact that the hybridization at the interface between the semiconductor states and the metal states for the majority spins is different from that of the minority spins $\left(\sim \boldsymbol{\Omega}_{s m}\right)$, is noncollinear to the magnetization of the FMM layer.

We demonstrated that the polarization of the electron spin density in the semiconductor layer, which is induced by the adjacent FMM layer, is oscillatory and has power-law falloff with the distance from the contact. The oscillatory behavior is characterized by the period $\lambda=\pi / \sqrt{2 m \mu}$ correlated with the position of the Fermi level as in the case of the ordinary Friedel oscillations. It is also interesting to note that the oscillations of $\mathbf{m}^{(\Lambda)}(z, \mu)$ and $\mathbf{m}^{(\Pi)}(z, \mu)$ are dephased by a quarter of period (with respect to each other).

\section{EFFECT OF THE FMM LAYER ON THE ORDERING OF LOCAL SPINS IN THE DMS LAYER}

Now, we consider the behavior of the local spins $\left\{\mathbf{S}_{i}\right\}$ dissolved in the DMS layer near its contact with the FMM layer. In FMM/DMS structures, the interface potential perturbs the spin and charge density of carriers in the semiconductor layer over rather large distances (on the order of the tunneling length) from the metal/semiconductor contact. This perturbation obviously affects the local spins dissolved in the DMS layer and influences their ordering. To describe this proximity effect in the framework of our model, we need to calculate the diagonal contribution $F_{d}$ to the free energy, Eq. (8). As it was already stated, we are assuming that the Fermi level of the system under consideration lies within the semiconductor conduction band near its edge. We can write $F_{d}$ in the explicit form

$$
F_{d}=\sum_{i} \operatorname{Im} \int^{\mu} \frac{d \omega}{\pi} \ln D\left(\mathbf{R}_{i}, \omega\right)
$$

with

$$
D\left(\mathbf{R}_{i}, \omega\right)=\operatorname{det}\left[\delta_{\alpha \beta}-\sum_{\gamma} g_{\alpha \gamma}\left(\mathbf{R}_{i}, \mathbf{R}_{i}, \omega\right) \kappa\left(\mathbf{S}_{i} \cdot \boldsymbol{\sigma}_{\gamma \beta}\right)\right] .
$$

The diagonal Green's function $g_{\alpha \gamma}\left(\mathbf{R}_{i}, \mathbf{R}_{i}, \omega\right)$ is thoroughly determined in Sec. III at the lowest nonvanishing order in the perturbations, Eqs. (3) and (5). Within such an approximation, with an accuracy up to the terms of the first order in the dimensionless parameter $\kappa S g(|\kappa S g| \ll 1)$, we obtain for the exchange part of the diagonal free energy the simple expression,

$$
F_{d}=\kappa \sum_{i} \mathbf{m}\left(\mathbf{R}_{i}\right) \cdot \mathbf{S}_{i},
$$

where $\mathbf{m}\left(\mathbf{R}_{i}\right)$ is the spin polarization of carriers at the $i$ th local-spin site of the DMS layer. The quantity $\mathbf{m}_{i}=\mathbf{m}(\mathbf{r}$ $\left.=\mathbf{R}_{i}\right)=\mathbf{m}\left(Z_{i}\right)$ is given by Eqs. (19)-(23).

Equation (28) states that, within the single-site approximation, the impurity moments inside the DMS layer near the FMM layer are magnetized under influence of the induced spin polarization of carriers. According to the results of Sec. III, the value $\mathbf{m}\left(\mathbf{R}_{i}\right)$ is proportional to the magnetization of the FMM layer. Therefore, the integral of effective exchange coupling between the local spin in the DMS layer and the magnetization of the FMM layer is defined in this approximation by the spin susceptibility of the carriers of the DMS at the local-spin site. This integral depends on the position of the Fermi level and on the distance between the local-spin site and the FMM/DMS interface.

To determine the exchange coupling between the local spins dispersed in the DMS layer, one has to calculate the off-diagonal contribution to the free energy, Eq. (8), $F_{\text {od }}$. Within the approximation adopted hereby $(|\kappa S g| \ll 1)$, by keeping the terms up to second order in $g^{\text {od }}$, we obtain the expression

$$
\begin{aligned}
F_{o d}= & -\sum_{i j} \operatorname{Im} \int^{\mu} \frac{d \omega}{2 \pi} \operatorname{Tr}\left[g^{o d} t g^{o d} t\right]= \\
& -\sum_{i j} \operatorname{Im} \int^{\mu} \frac{d \omega}{2 \pi} \sum_{\alpha \beta \gamma \tau} g_{\alpha \beta}\left(\mathbf{R}_{i}, \mathbf{R}_{j}, \omega\right) \\
& \times t_{\beta \tau}\left(\mathbf{R}_{j}, \omega\right) g_{\tau \gamma}\left(\mathbf{R}_{j}, \mathbf{R}_{i}, \omega\right) t_{\gamma \alpha}\left(\mathbf{R}_{i}, \omega\right) .
\end{aligned}
$$

Here, the summation over the local-spin site indices $i$ and $j$ is carried out for $i \neq j$. The off-diagonal Green's function $g^{\text {od }}$ entering Eq. (29) is given in Eqs. (9)-(11), if one sets $\mathbf{r}$ $=\mathbf{R}_{i}$ and $\mathbf{r}^{\prime}=\mathbf{R}_{j}, i \neq j$.

To second order in the expansion parameter $\kappa S g$, the energy of the indirect exchange interaction between local spins in the DMS layer takes the Heisenberg form

$$
F_{o d}=\sum_{i j}\left[B_{i j}^{(0)}+B_{i j}^{(\Lambda)}+B_{i j}^{(\Pi)}\right]\left(\mathbf{S}_{i} \cdot \mathbf{S}_{j}\right) .
$$

The exchange integral $B_{i j}^{(0)}$ expresses the usual coupling between the local spins dissolved in the bulk degenerated semiconductor (without accounting for the presence of the FMM layer), while the exchange integrals $B_{i j}^{(\Lambda)}$ and $B_{i j}^{(\Pi)}$ describe new channels for the indirect exchange interaction between the local spins, which appear due to electron excitations both without and with change in a symmetry of the states at the FMM/DMS contact, respectively.

The integral $B_{i j}^{(0)}$ depends only on the distance between the local-spin sites and has the standard the RKKY-type oscillatory behavior,

$$
B_{i j}^{(0)}=\frac{\kappa^{2}}{4 \pi m}\left(\frac{m a^{3}}{2 \pi}\right)^{2} \frac{\left(2 k_{F} R_{i j}\right) \cos \left(2 k_{F} R_{i j}\right)-\sin \left(2 k_{F} R_{i j}\right)}{R_{i j}^{4}},
$$

where $R_{i j}=\left|\mathbf{R}_{i}-\mathbf{R}_{j}\right|$. In the limit of very low density of carriers, when the inverse Fermi wave number is large compared to the average distance between local spins $2 k_{F} R_{i j}$ 
$\ll 1$ (the Zener case), this interaction is always ferromagnetic and given by

$$
B_{i j}^{(0)} \simeq-\frac{2 \kappa^{2} k_{F}^{3}}{3 \pi m R_{i j}}\left(\frac{m a^{3}}{2 \pi}\right)^{2} .
$$

The value $B_{i j}^{(\Lambda)}$ can be written as

$$
B_{i j}^{(\Lambda)}=-4 \kappa^{2} U_{s} \operatorname{Im} \int^{\mu} \frac{d \omega}{2 \pi} g^{0}\left(\mathbf{R}_{i} ; \mathbf{R}_{j} ; \omega\right) \widetilde{\Lambda}\left(\mathbf{R}_{j} ; \mathbf{R}_{i} ; \omega\right) .
$$

The expression for $B_{i j}^{(\Pi)}$ can be cast in the form

$$
\begin{aligned}
B_{i j}^{(\Pi)}= & -4 \kappa^{2} \operatorname{Im} \int^{\mu} \frac{d \omega}{2 \pi} g^{0}\left(\mathbf{R}_{i} ; \mathbf{R}_{j} ; \omega\right)\left\{\left[U_{s m} U_{m s}+\left(\boldsymbol{\Omega}_{s m} \cdot \Omega_{m s}\right)\right]\right. \\
& \times\left[\widetilde{\Pi}^{(+)}\left(\mathbf{R}_{j} ; \mathbf{R}_{i} ; \omega\right)+\widetilde{\Pi}^{(-)}\left(\mathbf{R}_{j} ; \mathbf{R}_{i} ; \omega\right)\right]-\left[U_{s m}\left(\mathbf{n} \cdot \boldsymbol{\Omega}_{m s}\right)\right. \\
& \left.\left.+U_{m s}\left(\mathbf{n} \cdot \boldsymbol{\Omega}_{s m}\right)\right]\left[\widetilde{\Pi}^{(+)}\left(\mathbf{R}_{j} ; \mathbf{R}_{i} ; \omega\right)-\widetilde{\Pi}^{(-)}\left(\mathbf{R}_{j} ; \mathbf{R}_{i} ; \omega\right)\right]\right\} .
\end{aligned}
$$

To further simplify the calculations we suppose that the average distance between magnetic impurities is much smaller than the characteristic range of the space variation in the carrier spin density in the DMS layer, i.e., the condition $2 k_{F} R_{i j} \ll 1$ is fulfilled. On the other hand, not to violate the limits of the effective-mass approximation, the condition $\sqrt{2 m W} R_{i j} \gg 1$ must hold true. It can be shown that in the functions $\widetilde{\Lambda}\left(\mathbf{r} ; \mathbf{r}^{\prime} ; \omega\right)$ and $\widetilde{\Pi}^{( \pm)}\left(\mathbf{r} ; \mathbf{r}^{\prime} ; \omega\right)$ the corrections to the local values $\tilde{\Lambda}(\mathbf{r} ; \mathbf{r} ; \omega)$ and $\widetilde{\Pi}^{( \pm)}(\mathbf{r} ; \mathbf{r} ; \omega)$, are of second order in the argument $R_{i j}$. Therefore, in Eqs. (31) and (32), one may substitute the values, Eqs. (16) and (17) [where $z \rightarrow Z$ $\left.=\left(Z_{i}+Z_{j}\right) / 2\right]$ for the off-diagonal functions $\widetilde{\Lambda}\left(\mathbf{R}_{j} ; \mathbf{R}_{i} ; \omega\right)$ and $\widetilde{\Pi}^{( \pm)}\left(\mathbf{R}_{j} ; \mathbf{R}_{i} ; \omega\right)$, respectively. Under these conditions, we arrive to the following expressions for the exchange integrals caused by the contact,

$$
\begin{aligned}
& B_{i j}^{(\Lambda)}=-4 \kappa^{2} U_{s}\left(\frac{m a^{3}}{2 \pi}\right)^{3} \frac{\mu}{R_{i j}}\left[\phi\left(2 k_{F} Z\right)+\frac{2 k_{F} R_{i j}}{3} \phi^{(1)}\left(2 k_{F} Z\right)\right], \\
& \phi^{(1)}(\xi)=\operatorname{ci}(\xi)-\frac{\sin \xi}{\xi}-\frac{2 \cos \xi}{\xi^{2}}+\frac{2 \sin \xi}{\xi^{3}}, \\
& B_{i j}^{(I))}= 4 \kappa^{2} U_{h}\left(\frac{m a^{3}}{2 \pi}\right)^{3} \frac{1}{R_{i j}}\left[\mu \varphi\left(2 k_{F} Z\right)-E_{0} \eta\left(2 \sqrt{2 m E_{0}} Z\right)\right. \\
&\left.-\mu \frac{2 k_{F} R_{i j}}{3} \varphi^{(1)}\left(2 k_{F} Z\right)\right], \\
& \varphi^{(1)}(\xi)=\operatorname{si}(\xi)+\frac{\cos \xi}{\xi}-\frac{2 \sin \xi}{\xi^{2}}+\frac{2(1-\cos \xi)}{\xi^{3}},
\end{aligned}
$$

$$
\begin{aligned}
U_{h}= & \frac{\sqrt{\tilde{m}} \widetilde{a}^{3}}{\sqrt{2 E_{0}}}\left\{U_{s m} U_{m s}+\left(\boldsymbol{\Omega}_{s m} \cdot \boldsymbol{\Omega}_{m s}\right)+\frac{\Delta}{2 E_{0}}\left[U_{s m}\left(\mathbf{n} \cdot \Omega_{m s}\right)\right.\right. \\
& \left.\left.+U_{m s}\left(\mathbf{n} \cdot \boldsymbol{\Omega}_{s m}\right)\right]\right\},
\end{aligned}
$$

where we keep the first order in the small parameter $\Delta / E_{0}$. In the square brackets in Eqs. (33) and (34), we keep terms proportional to the small parameter $2 k_{F} R_{i j}$, because they can become important in the vicinity the points where the functions $\phi\left(2 k_{F} Z\right)$, Eq. (21), and $\varphi\left(2 k_{F} Z\right)$, Eq. (24), vanish.

The behavior of the range function, which determines the exchange integrals in the DMS layer at different distances from the contact with the FMM layer, is shown in Fig. 1. It is noteworthy that the contact-induced exchange integrals (33) and (34) are proportional to the inverse of the separation between the local spins, $R_{i j}$, as in the case of a bulk DMS (in the absence of the FMM layer) characterized by an exchange integral (30).

Far from the interface, the asymptotic behavior of the exchange integrals (33) and (34) at $2 k_{F} Z \gg 1$ takes the form

$$
B_{i j}^{(\Lambda)} \simeq-\frac{\kappa^{2} U_{s}}{m}\left(\frac{m a^{3}}{2 \pi}\right)^{3} \frac{1}{R_{i j} Z^{2}}\left[\sin \left(2 k_{F} Z\right)-k_{F} R_{i j} \cos \left(2 k_{F} Z\right)\right],
$$

$$
B_{i j}^{(\Pi)} \simeq-\frac{\kappa^{2} U_{h}}{m}\left(\frac{m a^{3}}{2 \pi}\right)^{3} \frac{1}{R_{i j} Z^{2}}\left[\cos \left(2 k_{F} Z\right)-k_{F} R_{i j} \sin \left(2 k_{F} Z\right)\right] .
$$

One can see that the contact-induced exchange interaction decays with a power law when moving off the interface with the FMM layer and alternates its sign with a period $\sim \pi / k_{F}$, i.e., it can be either ferromagnetic or antiferromagnetic at different distances from the FMM/DMS interface. The oscillations of the integrals $B_{i j}^{(\Lambda)}$ and $B_{i j}^{(\Pi)}$ are dephased by a quarter of the period with respect to each other.

Comparing Eq. (30) with Eqs. (36) and (37), according to which the contact-induced exchange coupling among the local spins decreases as a power of a distance from the FMM/ DMS interface, $B_{i j}^{(\Lambda, \Pi)} \sim Z^{-2}$, while the magnitude of $B_{i j}^{(0)}$ does not depend on $Z$, we find that the ratio of their amplitudes does not exceed the value

$$
\frac{\left|B_{i j}^{(\Lambda, \Pi)}\right|}{\left|B_{i j}^{(0)}\right|} \approx \frac{\left(\left|U_{s}\right|,\left|U_{h}\right|\right) m a^{3}}{k_{F}^{3} Z^{2}} .
$$

In the region near the interface, the contact-induced exchange coupling has other features. Taking the limit $2 k_{F} Z$ $\ll 1$ and $2 \sqrt{2 m E_{0}} Z \ll 1$, we have

$$
B_{i j}^{(\Lambda)} \simeq-4 \kappa^{2} U_{s}\left(\frac{m a^{3}}{2 \pi}\right)^{3} \frac{\mu}{R_{i j}}\left[-\frac{\pi}{2}+\frac{4 k_{F} Z}{3}+\frac{2 k_{F} R_{i j}}{3} \ln \left(2 k_{F} Z\right)\right],
$$




$$
B_{i j}^{(\Pi)} \simeq 4 \kappa^{2} U_{h}\left(\frac{m a^{3}}{2 \pi}\right)^{3} \frac{1}{R_{i j}}\left[\mu \ln \left(2 k_{F} Z\right)-E_{0} \eta_{0}\right] .
$$

So, the value of the coupling increases slower than a power growth $\left(\sim Z^{-2}\right)$ when approaching the FMM/DMS interface.

The picture described above is applicable as long as the perturbation produced by the FMM/DMS interface on carriers and, consequently, on the local spins dissolved in the DMS layer, is small. Nevertheless, we can draw several important conclusions on how the FMM layer influences the exchange coupling between the local spins in the adjacent DMS layer. So, in proximity of the FMM/DMS interface, the interaction among the local spins dissolved in the DMS layer can be to some extent modified with respect to the bulk value due to the additional channels of the indirect coupling derived from the hybridization of the semiconductor quasiparticle states at the interface with the FMM layer. Whether the exchange coupling between the local spins will be enhanced or reduced, depends on the parameters of the contact potential $\left(U_{s}\right.$ and $\left.U_{h}\right)$ and on the distance from the interface $(Z)$. The contact-induced exchange interactions are long-range decaying and exhibit periodical switches from negative to positive as the distance from the interface varies. We emphasize the remarkable fact that it is possible to renormalize the exchange coupling in the DMS near the interface even with a nonmagnetic metal. Indeed, the exchange integrals $B_{i j}^{(\Lambda)}$ and $B_{i j}^{(\Pi)}$ do not vanish even in the case of $\boldsymbol{\Omega}_{s m}=0$ and $\boldsymbol{\Delta}=0$.

At fixed distance from the FMM/DMS interface, the sign of the integral $B_{i j}^{(\Lambda)}$ is related to the sign of the matrix element $U_{s}$, which, in turn, is determined by a charge redistribution between the semiconductor and the metal at the interface. Hence, one may easily see from Eq. (39), that the proximity to the FMM layer will result either in an enhancement of the ferromagnetic coupling among the local spins in the DMS layer (if $U_{s}<0$ ) or in a reduction (if $U_{s}>0$ ). The range function $\phi(\xi)$ shown in Fig. 1 makes this effect remarkable within a fairly broad region on the order of $k_{F} z$ $\lesssim 1-1.5$ near the interface. On the other hand, as it is clear from Eq. (38), in the framework of the perturbation expansion adopted in the interface matrix elements, the coupling component caused by the intraband processes at the interface is always considerably weaker than its bulk counterpart: $\left|B_{i j}^{(\Lambda)}\right| /\left|B_{i j}^{(0)}\right| \simeq\left(\left|U_{s}\right| a^{3} \sqrt{m} / \sqrt{\mu}\right) \ll 1$.

In the case of a nonmagnetic metal, at fixed distance from the interface, the sign of the integral $B_{i j}^{(\Pi)}$ is predetermined, since $U_{h}=\sqrt{\tilde{m}} \widetilde{a}^{3} U_{s m} U_{m s} / \sqrt{2 E_{0}}>0$. In this case, as seen from the behavior of the range function $\varphi(\xi)$, the interface hybridization results in an enhancement of the ferromagnetic coupling between the local spins in the DMS layer near the interface at $k_{F} z \lesssim 0.5$ where $\left|B_{i j}^{(\Pi)}\right| /\left|B_{i j}^{(0)}\right|$ $\simeq\left|U_{h}\right| a^{3} m \ln \left(2 k_{F} z\right) / k_{F}$. In the case of the contact with the FMM layer, the effective interface parameter $U_{h}$, Eq. (35), may be negative. Thus, we demonstrate that it is possible to enhance, reduce, and even reverse the exchange coupling in near-contact region of the DMS layer for various combinations of the constituent materials of the hybrid structure. It is interesting to note that, in contrast to the quantities $B_{i j}^{(0)}$ and $B_{i j}^{(\Lambda)}$, the exchange integral $B_{i j}^{(\Pi)}$ does not vanish even if the carrier concentration in the semiconductor layer tends to zero, i.e., at $\mu \rightarrow 0$. Under the condition that $\left|\mu \ln \left(2 k_{F} z\right)\right|$ $<E_{0}$, the interaction between the local spins in the DMS layer is mainly determined by quasiparticle excitations through the metal states with negative energies, $\omega<0$. Thanks to these interface processes, provided the value $\tilde{U}$ is small, in virtue of the relatively sizable density of states at the Fermi level in the metal, $N_{m}(\omega=\mu) \propto \sqrt{E_{0} \tilde{m}^{3}}$, the exchange integral $B_{i j}^{(\Pi)}$, Eq. (40), could turn to be comparable with (or larger than) the inherent exchange integral of DMS $B_{i j}^{(0)}$, Eq. (30), in the narrow near-contact region on the order of the tunneling length $\left(\sqrt{2 m E_{0}}\right)^{-1}$.

\section{MEAN-FIELD DESCRIPTION OF MAGNETIC ORDERING IN THE DMS LAYER NEAR THE FMM/DMS INTERFACE}

Above we have demonstrated that it is possible to enhance or reduce the indirect exchange coupling between local spins in the DMS layer in the proximity of the FMM layer. This supports the idea that the magnetic properties of the DMS layer near the FMM/DMS interface could be different from the bulk properties of DMS.

According to our foregoing microscopic considerations, the whole expression for the exchange energy of local spins in the DMS layer in contact with the FMM layer can be cast in the form

$$
F^{(e x)}=\kappa \sum_{i}\left(\mathbf{m}_{i} \cdot \mathbf{S}_{i}\right)+\sum_{i j}\left[B_{i j}^{(0)}+B_{i j}^{(\Lambda)}+B_{i j}^{(\Pi)}\right]\left(\mathbf{S}_{i} \cdot \mathbf{S}_{j}\right) .
$$

It is important to emphasize that all components of the carrier-mediated exchange coupling involved in Eq. (41) are essentially constrained in sign as functions of the distance between local spins, except for large $R_{i j} \geq k_{F}^{-1}$, and therefore, as long as the carrier density $n \sim k_{F}^{3}$ is low, $n \ll n_{m}$, where $n_{m}$ is the density of the local spins (i.e., typical $R_{i j} \sim n_{m}^{-1 / 3}$ ), the frustration effects associated with the oscillatory nature of the RKKY-type mechanism are immaterial. Thus, carrying out the double summation in Eq. (41), we retain the paired links only between the nearest-neighboring local-spin sites, $\langle i j\rangle$, and apply Eqs. (30), (33), and (34) for the exchange integrals $B_{i j}^{(0)}, B_{i j}^{(\Lambda)}$, and $B_{i j}^{(\Pi)}$, respectively.

In this section, we develop a mean-field description for the magnetic ordering in the DMS layer in contact with the FMM layer. We take into account both the bulk and contactinduced mechanisms of indirect exchange between the local spins as well as the contact-induced spin polarization of carriers in the DMS layer. Note that the Weiss mean-field theory for the bulk DMS developed in Ref. 18 yields long-range FM ordering of the local spins below the mean-field Curie temperature $T_{c}^{(M F)} \sim n_{m} n^{1 / 3}$ for all values of $n$ and $n_{m}$. Following the mean-field approach, let us write an effective mean-field Hamiltonian $H_{\text {eff }}^{(e x)}$ of the system. It can be obtained replacing of the random exchange field of the local spins $\mathbf{S}_{i}$ inside the DMS layer by its averaged density using the standard substitution $\mathbf{S}_{i} \rightarrow\left\langle\mathbf{S}_{i}\right\rangle$, where the angular brackets denote averaging over the configurations of local spin. It is necessary to pay special attention to the sense of this averaging procedure in 
our situation. Even if the dopant concentration is assumed perfectly homogeneous in the DMS layer along the $z$ axis, the averaged spin density $\left\langle\mathbf{S}_{i}\right\rangle$ is inhomogeneous, implying that both the spin polarization of the carriers and the contact induced component of the exchange integral are varying along the $z$ direction. So far as we assume that the local spins in the DMS layer are randomly distributed and dense on a scale set by the Fermi wave vector of the carriers $\lambda=\pi / k_{F}$, i.e., $n=k_{F}^{3} /\left(3 \pi^{2}\right) \ll n_{m}$, the classical continuous picture of the exchange field, provided by these spins, seems reasonable. This allows us to adopt a continuum approximation, where the averaged spin density $\langle\mathbf{S}\rangle(z)$ depends only on the $z$ coordinate, $\left\langle\mathbf{S}_{i}\right\rangle=\langle\mathbf{S}\rangle\left(Z_{i}\right)$, and from Eq. (41) one can obtain

$$
\begin{gathered}
F_{e f f}^{(e x)}=\sum_{i} g \mu_{B}\left(\mathbf{b}_{i} \cdot \mathbf{S}_{i}\right), \\
g \mu_{B} \mathbf{b}_{i}=\kappa \mathbf{m}_{i}+\left[B^{(0)}+B_{i}^{(\Lambda)}+B_{i}^{(\Pi)}\right]\left\langle\mathbf{S}_{i}\right\rangle .
\end{gathered}
$$

Here, $\mathbf{b}_{i}$ is the effective exchange field, seen by the local spin $\mathbf{S}_{i}, g$ is the $g$ factor corresponding to the local spin, $\mu_{B}$ is the Bohr magneton. We retain the site dependence in $\mathbf{b}_{i}$, thereby taking into account the contact influence, which subjects different local spins to different exchange couplings depending on the local-spin position. Within the framework of our model, the effective exchange field inside the DMS layer depends only on the distance along the $z$ direction between the local-spin site and the FMM/DMS interface, $\mathbf{b}_{i}=\mathbf{b}\left(Z_{i}\right)$.

The first term in $\mathbf{b}_{i}$, Eq. (42), describes the built-in field of the spin polarization of the carriers induced in the DMS layer by the contact with the FMM layer. The second term in $\mathbf{b}_{i}$, Eq. (42), contains different components of the Weiss mean field arising from the indirect exchange coupling between the local spins, where $B_{i}^{(0, \Lambda, \Pi)}=\Sigma_{j} B_{i j}^{(0, \Lambda, \Pi)}$ is the sum of all links to the nearest local spins surrounding the site $i$. One can make the rough estimates

$$
\begin{aligned}
& B^{(0)}=B_{i}^{(0)} \simeq 4 \pi \mathcal{Z} n_{m} \int_{R_{A}}^{R_{0}} d R R^{2} B^{(0)}(R), \\
& B_{i}^{(\Lambda, \Pi)} \simeq 4 \pi \mathcal{Z} n_{m} \int_{R_{A}}^{R_{0}} d R R^{2} B^{(\Lambda, \Pi)}\left(R, Z_{i}\right),
\end{aligned}
$$

where $\mathcal{Z}$ is the average coordination number for an ion carrying the local spin. In Eqs. (43) and (44), the integration over the difference variable $R=R_{i j}$ is restricted to a finite interval. Taking a nonzero lower limit $R_{A}$, we avoid necessity to introduce explicitly into our model an additional superexchange coupling between the nearest-neighbor local spins. This coupling in DMS is, as a rule, antiferromagnetic and short ranged with a radius $\approx R_{A} \cdot{ }^{19}$ The upper limit of the integration $R_{0}$ is associated with a finite carrier mean-free path due to scattering effects in the alloy that could be explicitly incorporated into the theory whenever needed. In this case, the exchange integrals $B_{i j}^{(0, \Lambda, \Pi)}$ should acquire an exponential prefactor $\exp \left(-R_{i j} / R_{0}\right) .{ }^{i j}$

Assuming that the local spins are in equilibrium with the thermal bath and the semiconductor is degenerated, $\mu \gg T$, the mean-field free energy reads $F_{M F}^{(e x)}=-k_{B} T \ln \operatorname{tr} \exp ($ $\left.-H_{e f f}^{(e x)} / k_{B} T\right)$. Minimizing $F_{M F}^{(e x)}$ with respect to the order pa- rameter $\left\langle\mathbf{S}_{i}\right\rangle$, we obtain the self-consistency equation for the averaged spin density at the site $i$ in the effective field $\mathbf{b}_{i}$, Eq. (42), in the absence of external fields,

$$
\left\langle\mathbf{S}_{i}\right\rangle=\operatorname{Sn} \mathcal{L}\left(S \frac{g \mu_{B}\left|\mathbf{b}_{i}\right|}{k_{B} T}\right),
$$

where $\mathbf{n}$ is the unit vector parallel to the field $\mathbf{b}_{i}, \mathcal{L}(b)$ is the Langevin function, and $k_{B}$ is the Boltzmann constant. Now, the average of the spin density determined by Eq. (45) is understood as a thermal average and an average with respect to disorder.

Primarily, we address the case when $\mathbf{m}_{i}=0$ in Eq. (42). By expanding Eq. (45) for small $\left\langle\mathbf{S}_{i}\right\rangle$, we find the averaged value of the local spin in the DMS layer in the vicinity of the ferromagnetic transition

$$
\frac{\langle S\rangle(z)}{S}=\sqrt{\frac{5}{3} \frac{T_{c}(z)-T}{T_{c}(z)}} .
$$

The critical temperature is expressed as

$$
T_{c}(z)=T_{c}^{(0)}-\frac{S^{2}}{3 k_{B}}\left[B^{(\Lambda)}(z)+B^{(\Pi)}(z)\right],
$$

where

$$
T_{c}^{(0)}=\frac{S^{2}}{3 k_{B}}\left|B^{(0)}\right|
$$

is the Curie temperature in the bulk DMS since the exchange integral $B^{(0)}$ gives rise to uniform FM. One can see that, within the framework of the classical approach, the intrinsic critical temperature $T_{c}(z)$ corresponds to ferromagnetic ordering in the plane inside the DMS layer that is at the distance $z$ measured from the interface, and the difference $T_{c}(z)-T_{c}^{(0)}$ follows the long-range space variation in the contact-induced exchange integrals. It is clear that above the transition temperature, at $T>T_{c}(z)$, the order parameter vanishes, $\langle\mathbf{S}\rangle=0$, while at low temperature it becomes saturated, $\langle\mathbf{S}\rangle \rightarrow S$. At large distances from the interface $z \gg k_{F}^{-1}$, the dependence $\langle\mathbf{S}\rangle(z)$ approaches the value $\langle\mathbf{S}\rangle^{(0)}$ in the bulk DMS.

The solutions of Eq. (45) for the order parameter $\langle\mathbf{S}\rangle(z)$ at the temperature $T$ may be obtained numerically. We illustrate our findings for several particular situations in Figs. 2-4, where the variables are expressed in the dimensionless units $\tilde{z}=2 k_{F} z$ and $t=T / T_{c}^{(0)}$. Within this choice, the value $t=1$ corresponds to the Curie temperature of the bulk DMS $T_{c}^{(0)}, \mathrm{Eq}$. (46).

Under the assumption that $\boldsymbol{\Omega}_{s}=\boldsymbol{\Omega}_{h}=0$ and $U_{h}=0$, in other words $m_{i}=0$ and $B_{i}^{(\Pi)}=0$ in Eq. (42), the contact-induced variation in $\langle\mathbf{S}\rangle(z)$ is due to the spin-independent carrier scattering at the interface. In Fig. 2 we show the relevant spatial profile $\langle\mathbf{S}\rangle(z, T)$ at different temperatures for $U_{s}<0$ with $\left|u_{s}\right|=3\left|U_{s}\right| m a^{3} /\left(2 k_{F}\right)=0.5$. This value is chosen, on the one hand, to emphasize the contact effect and, on the other hand, not go too far beyond the limits of applicability of our approach. 


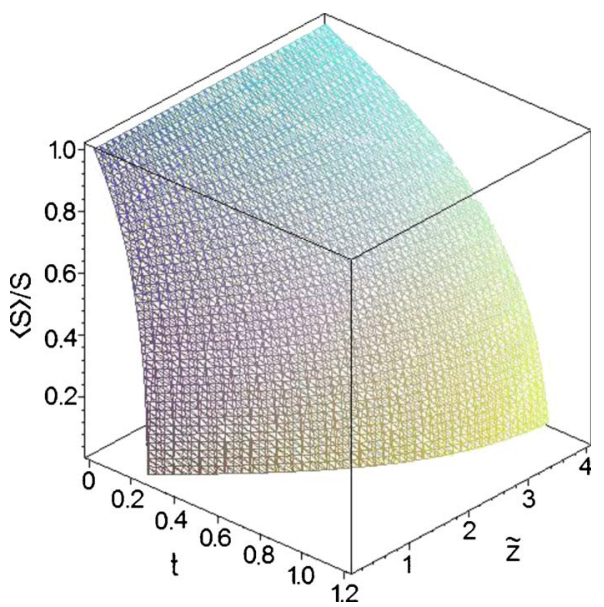

FIG. 2. (Color online) Average value of the local spin as a function of the dimensionless distance from the interface, $\widetilde{z}=2 k_{F} z$ and dimensionless temperature $t=T / T_{c}^{(0)}$, for $\boldsymbol{\Omega}_{s}=\boldsymbol{\Omega}_{h}=0, U_{h}=0$, and $U_{s}<0$ with $\left|u_{s}\right|=3\left|U_{s}\right| m a^{3} /\left(2 k_{F}\right)=0.5$.

Under the assumption that $\boldsymbol{\Omega}_{s}=\boldsymbol{\Omega}_{h}=0$ and $U_{s}=0$, in other words $m_{i}=0$ and $B_{i}^{(\Lambda)}=0$ in Eq. (42), the contact-induced variation in $\langle\mathbf{S}\rangle(z)$ is only due to the hybridization of the semiconductor and metal states at the interface. In Fig. 3 we show the relevant spatial profile $\langle\mathbf{S}\rangle(z, T)$ at different temperatures for $U_{h}>0$ with $u_{h}=3 U_{h} m a^{3} /\left(2 k_{F}\right)=0.1, \mu / E_{0}$ $=1 / 9$, and $m / \widetilde{m}=1 / 5$.

Our results show that the averaged value of the local spin in the DMS layer increases (decreases) when approaching the interface with the metal, and this is interpreted as a consequence of the enhanced (suppressed) exchange coupling, due to the presence of the metal layer. The contact-induced variation in $\langle\mathbf{S}\rangle(z)$ extends over a significant length scale on the order of the Fermi wavelength. The DMS layer juxtaposed to the metal layer can exhibit ferromagnetic ordering of the local spins even at elevated temperatures where no such ordering exists in the corresponding bulk material.

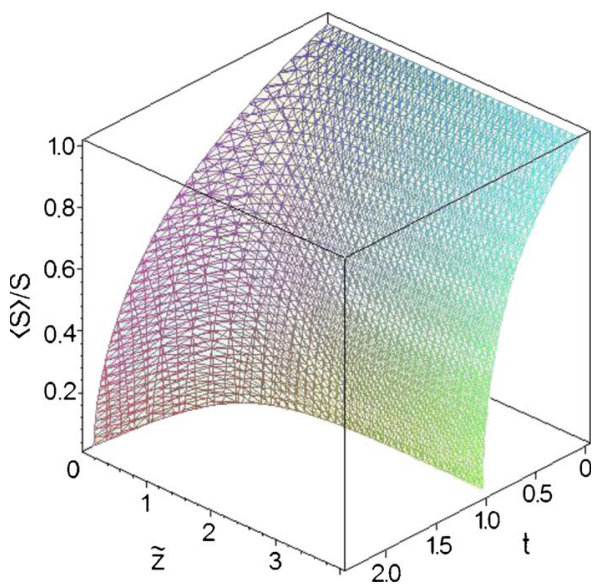

FIG. 3. (Color online) Average value of the local spin as a function of the dimensionless distance from the interface, $\tilde{z}=2 k_{F} z$. and dimensionless temperature $t=T / T_{c}^{(0)}$, for $\boldsymbol{\Omega}_{s}=\boldsymbol{\Omega}_{h}=0, U_{s}=0$, and $U_{h}>0$ with $u_{h}=3 U_{h} m a^{3} /\left(2 k_{F}\right)=0.1, \mu / E_{0}=1 / 9$, and $m / \tilde{m}$ $=1 / 5$.



FIG. 4. Average value of the local spin as a function of the dimensionless distance from the interface, $\tilde{z}=2 k_{F} z$ and dimensionless temperature $t=T / T_{c}^{(0)}$, for $U_{s}=U_{h}=0, \quad \boldsymbol{\Omega}_{h}=0$, and $\left|\gamma_{s}\right|$ $=\left|\kappa \boldsymbol{\Omega}_{s}\right| \mu\left(m a^{3} / \pi\right)^{2} /\left(S\left|B^{(0)}\right|\right)=0.5$.

In the case when $\mathbf{m}_{i} \neq 0$, well above the critical temperature $T_{c}(z)$, the averaged value of the local spin is described by the formula

$$
\langle\mathbf{S}\rangle(z)=-\frac{S^{2} \kappa \mathbf{m}(z)}{3 k_{B}\left[T-T_{c}(z)\right]} .
$$

If one neglects the dependence of the critical temperature $T_{c}$ on the distance $z$, the net magnetization of the DMS layer $\mathbf{M}=\int_{0}^{\infty} d z\langle\mathbf{S}\rangle(z)$ is proportional to the net spin polarization of carriers $\boldsymbol{\nu}$, Eq. (27).

Under the assumption of $U_{s}=U_{h}=0$, in other words $B_{i}^{(\Lambda)}$ $=0$ and $B_{i}^{(\Pi)}=0$ in Eq. (42), the contact-induced variation in $\langle\mathbf{S}\rangle(z)$ is caused by the carrier polarization $\mathbf{m}(z)$ which is influenced by the contact. The value of $\langle\mathbf{S}\rangle(z)$ as a function of the distance $z$ and temperature $T$ at $\boldsymbol{\Omega}_{h}=0$ and $\left|\gamma_{s}\right|=0.5$ is shown in Fig. 4. It is implied that the vector $\langle\mathbf{S}\rangle(z)$ is strictly parallel (antiparallel) to the vector $\mathbf{m}(z)$ if $\kappa<0 \quad(\kappa>0)$. Here, we introduce the dimensionless interaction parameter $\gamma_{s}=\kappa\left|\Omega_{s}\right| \mu\left(m a^{3} / \pi\right)^{2} /\left(S\left|B^{(0)}\right|\right)$ which, unlike the parameters $u_{s}$ and $u_{h}$, is inversely proportional to the concentration of the local spins $n_{m}$. We draw the inference that, in the DMS layer juxtaposed to the FMM layer, the contact-induced ferromagnetic ordering of the local spins survives at temperatures much larger than the value in the bulk DMS, $T_{c}^{(0)}$.

\section{SUMMARY AND CONCLUDING REMARKS}

The approach discussed above enables us to capture the delicate interfacial process playing a key role in determining the magnetic properties of the FMM/DMS structures. We carried out the systematic investigation of the conditions for the appearance of the spin polarization of carriers and the ferromagnetic ordering of the local spins in the DMS layer by contacting it to the FMM layer. Our analysis provides insight into the microscopic mechanism of the proximity effect in the semiconductor/metal hybrid structure. The nature of the proximity effect is intricate enough. One has to distin- 
guish two different ways for modifying the ordering of the local spins in the DMS layer. First, the FMM layer magnetizes the local spins diluted in the semiconductor through the induced spin polarization of carriers. Second, the carriermediated interaction between the local spins changes near the interface with the metal due to the additional channel of the exchange coupling via the metal layer. It should be noted that the second effect works even in the case of nonmagnetic metal layer. The magnitude of the effect depends on the distance from the interface as well as on the position of the Fermi level in the semiconductor.

Our theory demonstrated that high-temperature magnetic ordering in the DMS layer can be controlled by the electron processes at the interface with the FMM layer. On the basis of our results, we can offer qualitative explanations for a number of curious features, experimentally observed on the $\mathrm{Fe} /(\mathrm{Ga}, \mathrm{Mn}) \mathrm{As}$ (Refs. 11 and 21) and Py/(Ga,Mn)As (Ref. 15) hybrid systems, that have attracted great interest.

In the Ref. 11, Maccherozzi et al. report X-ray magnetic circular dichroism and superconducting quantum interference device (SQUID) magnetometry experiments to study magnetic order and coupling in thin $\mathrm{Fe} /(\mathrm{Ga}, \mathrm{Mn}) \mathrm{As}(100)$ films, which are grown by means of molecular-beam epitaxy. They observed induced magnetic order in the (Ga,Mn)As layer that persists up to room temperature, for various $\mathrm{Mn}$ concentrations and $\mathrm{Fe}$ thicknesses, and extends over the (Ga,Mn)As region as thick as $2 \mathrm{~nm}$. In fact, up to $T=60 \mathrm{~K}$, the SQUID measurements display the spontaneous magnetization curves of bulk ferromagnetic (Ga,Mn)As offset by the Fe magnetization, whereas for $T>60 \mathrm{~K}$ the magnetization in $(\mathrm{Ga}, \mathrm{Mn}) \mathrm{As}$ is entirely due to the Fe film, whose Curie temperature is well above room temperature. This indicates that magnetic ordering can be induced in the DMS layer when contacted to the magnetic metal layer. The above developed model supplies the possible way to explain the mechanism of this proximity phenomenon. According to Eq. (47), well above the critical temperature of the bulk DMS, $T_{c}^{(0)}$, the magnetization of the local spins in the DMS layer, $\mathbf{M}(T)$, which is proportional to the averaged value of the local spin, $\langle\mathbf{S}\rangle(z)$, is bound to the magnetization of the FMM layer, $\sim \boldsymbol{\Delta}$, via the induced spin polarization of carriers, $\mathbf{m}(z) \sim \boldsymbol{\Delta}$ [Eqs. (20) and (22)], and extends in the nearinterface region, over a length scale which is on the order of the Fermi wavelength. It is easy to estimate that in our model the characteristic length of induced ferromagnetic ordering may achieve $\sim 2 \mathrm{~nm}$ (as in Ref. 11) for carrier concentrations between $10^{20}$ and $10^{21} \mathrm{~cm}^{-3}$.

It is worth to notice that, on the one hand, the observed Curie temperature (of appearance of the FM long-range order) is not affected by the Fe overlayer and has the same $T_{c}$ as the pure $(\mathrm{Ga}, \mathrm{Mn})$ As substrate, i.e., $T_{c}=T_{c}^{(0)}$, on the other hand, at $T>T_{c}^{(0)}$ the magnetization of the $(\mathrm{Ga}, \mathrm{Mn})$ As layer, induced by the presence of the Fe overlayer (the FM shortrange order), is rather large, e.g., the value $M(T=200 \mathrm{~K})$ accounts for $\approx 20 \%$ of the maximum $M(T=0 \mathrm{~K}) .{ }^{11}$ Therefore, we can consider the ordering of the local spins in the DMS layer caused by the spin polarization of carriers near the FMM/DMS contact as the dominant mechanism governing the proximity effect in the $\mathrm{Fe} /(\mathrm{Ga}, \mathrm{Mn}) \mathrm{As}(100)$ structure. Under the conditions $U_{s}=U_{h}=0$, taking into account Eqs.



FIG. 5. (Color online) Comparison of the theoretical results with experimental data. The plot contains the experimental $M(T)$ data from Ref. 11 (ovals) and the theoretical curve computed from Eq. (48) (thick line). The magnetization and temperature are represented through the dimensionless variables $M / M(0)=M(T) / M(T=0)$ and $t=T / T_{c}^{(0)}$, respectively.

(27) and (47), our theory allows to predict the Curie-Weiss temperature dependence of the net magnetization of the DMS layer at elevated temperatures, $T \gg T_{c}^{(0)}$,

$$
\mathbf{M}(T)=-\frac{S^{2} \kappa \boldsymbol{\nu}}{3 k_{B}\left[T-T_{c}^{(0)}\right]} .
$$

It is instructive to compare this result with experimental data since this comparison provides valuable estimates for the parameters of the interface potential responsible for the ordering in the DMS layer contacted to the FMM layer. In Fig. 5 the temperature dependence, Eq. (47), is superimposed to the experimental curve of the spontaneous magnetization of the DMS layer in a Fe/(Ga,Mn)As(100) sample with $6 \% \mathrm{Mn}$ concentration and bulk critical temperature $T_{c}^{(0)} \simeq 62 \mathrm{~K}$. One can see that the theoretical dependence is, at least qualitatively, compatible with the experimental findings in a wide temperature range, if one takes $\kappa|\nu| S /\left(3 k_{B} T_{c}^{(0)} L\right)=0.35(L$ is the thickness of the DMS layer, $\left.L>\pi k_{F}^{-1}\right)$. The estimate for the model parameters which determine the asymptotic behavior of $M(T)$ can be rewritten in the following form: $\left|\gamma_{s}\right| \simeq 0.35 k_{F} L$ or $\left|\gamma_{h}\right| \simeq 0.35 k_{F} L$, where

$$
\left|\gamma_{s}\right|=\left|\kappa \Omega_{s}\right| \mu\left(m a^{3} / \pi\right)^{2} /\left(S|B|^{(0)}\right)
$$

or

$$
\left|\gamma_{h}\right|=\left|\kappa \boldsymbol{\Omega}_{h}\right| \sqrt{\mu E_{0}}\left(m a^{3} / \pi\right)^{2} /\left(S|B|^{(0)}\right)
$$

[equivalently, $S a^{2}\left|\kappa \Omega_{s}\right| \sqrt{\mu / m} / L \approx\left(10^{-2}-10^{-3}\right) \mathrm{eV}^{3} \quad$ or $\left.S a^{2}{ }_{\kappa} \Omega_{h} \mid \sqrt{E_{0} / m} / L \approx\left(10^{-2}-10^{-3}\right) \mathrm{eV}^{3}\right]$. Note that the parameters $\left|\gamma_{s}\right|$ and $\left|\gamma_{h}\right|$ depend differently on the concentration $n$ in the DMS.

Another attempt to combine two different ferromagnetic materials, a semiconductor $[(\mathrm{Ga}, \mathrm{Mn}) \mathrm{As}]$ and a metal (permalloy), was undertaken in Ref. 15. Mark and co-workers demonstrated that the coupling of the DMS layer to the FMM layer observed in Ref. 11 does not preclude independent magnetic behavior of the ferromagnetic ( $\mathrm{Ga}, \mathrm{Mn}) \mathrm{As}$ and permalloy epilayers, when these are directly deposited on each other, without the need for a nonmagnetic intermediate 
layer. When these layers are in direct contact, their magnetization can still be switched independently, as it is confirmed both by transport observations, and direct magnetization measurements using SQUID. In the framework of our model, the lack of magnetic coupling between the $(\mathrm{Ga}, \mathrm{Mn}) \mathrm{As}$ and permalloy layers could be explained by assuming, for example, a charge redistribution between the semiconductor and the metal at the interface such that the corresponding potential is positive, $U_{s}>0$. As noted in Sec. IV and illustrated in Fig. 3, this results in a reduction in the interaction among the local spins and, hence, ferromagnetic ordering within a fairly broad region in the DMS near the interface. The same consequences might be caused by strong scattering of carriers at imperfections of the $\mathrm{Py} /(\mathrm{Ga}, \mathrm{Mn}) \mathrm{As}$ interface, such as roughness and/or interdiffusion of atomic species, which can never be fully excluded.

In conclusion, we proposed a semiphenomenological model to describe the spin ordering in a DMS layer contacted with a FMM layer. We analyzed two types of proximity effects: (1) the ordering of the local spins in a DMS induced by the spin polarization of carriers near the FMM/
DMS contact; (2) the indirect exchange coupling among the local spins in a DMS provided by the variation in the spin susceptibility of the carriers near the FMM/DMS contact. Both effects owe their appearance to the hybridization of the metal and semiconductor electron states at the interface. Of course, the current model is too simple to account for all aspects of the phenomenon. We hope that the results presented in this paper will stimulate further theoretical and experimental investigation on this intricate subject.

\section{ACKNOWLEDGMENTS}

The work was partially supported by the University of the Basque Country [Proyecto GV-UPV/EHU under Grant No. IT-366-07), Spanish Ministerio de Ciencia y Tecnología (Grant No. FIS2007-66711-C02-01)], and by RFBR (Grant No. 10-02-00118). S.C. also acknowledges financial support by PRIN 2007 under Project No. 2007FW3MJX003. V.V.T. acknowledges financial support by Ikerbasque (Basque Foundation for Science).
${ }^{1}$ T. Jungwirth, J. Sinova, J. Mašek, J. Kučera, and A. H. MacDonald, Rev. Mod. Phys. 78, 809 (2006).

${ }^{2}$ K. C. Ku, S. J. Potashnik, R. F. Wang, S. H. Chun, P. Schiffer, N. Samarth, M. J. Seong, A. Mascarenhas, E. Johnston-Halperin, R. C. Myers, A. C. Gossard, and D. D. Awschalom, Appl. Phys. Lett. 82, 2302 (2003).

${ }^{3}$ A. P. Li, J. Shen, J. R. Thompson, and H. H. Weitering, Appl. Phys. Lett. 86, 152507 (2005); A. P. Li, J. F. Wendelken, J. Shen, L. C. Feldman, J. R. Thompson, and H. H. Weitering, Phys. Rev. B 72, 195205 (2005).

${ }^{4}$ S. Sanvito and N. A. Hill, Phys. Rev. Lett. 87, 267202 (2001).

${ }^{5}$ M. C. Qian, C. Y. Fong, W. E. Pickett, J. E. Pask, L. H. Yang, and S. Dag, Phys. Rev. B 71, 012414 (2005).

${ }^{6}$ A. Continenza, F. Antoniella, and S. Picozzi, Phys. Rev. B 70, 035310 (2004).

${ }^{7}$ V. N. Men'shov, V. V. Tugushev, P. M. Echenique, S. Caprara, and E. V. Chulkov, Phys. Rev. B 78, 024438 (2008).

${ }^{8}$ V. N. Men'shov, V. V. Tugushev, S. Caprara, P. M. Echenique, and E. V. Chulkov, Phys. Rev. B 80, 035315 (2009).

${ }^{9}$ S. Caprara, V. V. Tugushev, P. M. Echenique, and E. V. Chulkov, EPL 85, 27006 (2009).

${ }^{10}$ A. M. Nazmul, T. Amemiya, Y. Shuto, S. Sugahara, and M. Tanaka, Phys. Rev. Lett. 95, 017201 (2005).

${ }^{11}$ F. Maccherozzi, M. Sperl, G. Panaccione, J. Minár, S. Polesya, H. Ebert, U. Wurstbauer, M. Hochstrasser, G. Rossi, G. Woltersdorf, W. Wegscheider, and C. H. Back, Phys. Rev. Lett. 101,
267201 (2008); M. Sperl, F. Maccherozzi, F. Borgatti, A. Verna, G. Rossi, M. Soda, D. Schuh, G. Bayreuther, W. Wegscheider, J. C. Cezar, F. Yakhou, N. B. Brookes, C. H. Back, and G. Panaccione, Phys. Rev. B 81, 035211 (2010).

${ }^{12} \mathrm{~S}$. Honda, H. Itoh, J. Inoue, H. Kurebayashi, T. Trypiniotis, C. H. W. Barnes, A. Hirohata, and J. A. C. Bland, Phys. Rev. B 78, 245316 (2008).

${ }^{13}$ J. C. Slonczewski, Phys. Rev. B 39, 6995 (1989).

${ }^{14}$ P. Bruno, Phys. Rev. B 52, 411 (1995).

${ }^{15}$ S. Mark, C. Gould, K. Pappert, J. Wenisch, K. Brunner, G. Schmidt, and L. W. Molenkamp, Phys. Rev. Lett. 103, 017204 (2009).

${ }^{16}$ J. Inoue, S. Nonoyama, and H. Itoh, Phys. Rev. Lett. 85, 4610 (2000); J. Inoue, Phys. Rev. B 67, 125302 (2003).

${ }^{17}$ Handbook of Mathematical Functions, edited by M. Abramowitz and I. A. Stegun (National Bureau of Standards, New York, 1964).

${ }^{18}$ T. Dietl, H. Ohno, and F. Matsukura, Phys. Rev. B 63, 195205 (2001).

${ }^{19}$ V. Barzykin, Phys. Rev. B 71, 155203 (2005).

${ }^{20}$ D. J. Priour, Jr., E. H. Hwang, and S. Das Sarma, Phys. Rev. Lett. 92, 117201 (2004).

${ }^{21}$ K. Olejnik, P. Wadley, J. A. Haigh, K. W. Edmonds, R. P. Campion, A. W. Rushforth, B. L. Gallagher, C. T. Foxon, T. Jungwirth, J. Wunderlich, S. S. Dhesi, S. A. Cavill, G. van der Laan, and E. Arenholz, Phys. Rev. B 81, 104402 (2010). 